Article

\title{
An Affine Model of a Riemann Surface Associated to a Schwarz-Christoffel Mapping
}

\author{
Richard Cushman
}

check for updates

Citation: Cushman, R. An Affine Model of a Riemann Surface Associated to a Schwarz-Christoffel Mapping. Axioms 2021, 10, 49. https://doi.org/10.3390/ axioms10020049

Academic Editor: Anna Maria Fino

Received: 19 January 2021

Accepted: 23 March 2021

Published: 1 April 2021

Publisher's Note: MDPI stays neutral with regard to jurisdictional claims in published maps and institutional affiliations.

Copyright: (C) 2021 by the author. Licensee MDPI, Basel, Switzerland. This article is an open access article distributed under the terms and conditions of the Creative Commons Attribution (CC BY) license (https:// creativecommons.org/licenses/by/ $4.0 /)$.
Department of Mathematics and Statistics, University of Calgary, Calgary, AB T2N 1N4, Canada; r.h.cushman@gmail.com

\begin{abstract}
In this paper, we construct an affine model of a Riemann surface with a flat Riemannian metric associated to a Schwarz-Christoffel mapping of the upper half plane onto a rational triangle. We explain the relation between the geodesics on this Riemann surface and billiard motions in a regular stellated $n$-gon in the complex plane.
\end{abstract}

Keywords: Schwarz-Christofell; Rieman surface; discrete subgroup

MSC: 30C30; 30F10

\section{Introduction}

We give a section by section summary of the contents of this paper.

In $\S 1$ we define the Schwarz-Christoffel conformal map $F_{Q}(2)$ of the complex plane less $\{0,1\}$ onto a quadrilateral $Q$, which is formed by reflecting a rational triangle $T_{n_{0} n_{1} n_{\infty}}$ in the real axis.

In $\S 2$, following Aurell and Itzykson [1] we associate to the map $F_{Q}$ the affine Riemann surface $\mathcal{S}$ in $\mathbb{C}^{2}$ defined by $\eta^{n}=\xi^{n-n_{0}}(1-\xi)^{n-n_{1}}$, where $\mathbb{C}^{2}$ has coordinates $(\xi, \eta)$ and $n=n_{0}+n_{1}+n_{\infty}$. Thinking of $\mathcal{S}$ as a branched covering

$$
\pi: \mathcal{S} \rightarrow \mathbb{C} \backslash\{0,1\}:(\xi, \eta) \mapsto \xi
$$

with branch points at $(0,0),(1,0)$ and $\infty$ corresponding to the branch values 0,1 , and $\infty$, respectively, we show that $\mathcal{S}$ has genus $\frac{1}{2}\left(n+2-\left(d_{0}+d_{1}+d_{\infty}\right)\right)$, where $d_{j}=\operatorname{gcd}\left(n, n_{j}\right)$ for $j=0,1, \infty$. Let $\mathcal{S}_{\text {reg }}$ be the set of nonsingular points of $\mathcal{S}$. The map $\widehat{\pi}=\pi_{\mid \mathcal{S}_{\text {reg }}}: \mathcal{S}_{\text {reg }} \rightarrow$ $\mathbb{C} \backslash\{0,1\}$ is a holomorphic $n$-fold covering map with covering group the cyclic group generated by

$$
\mathcal{R}: \mathcal{S}_{\text {reg }} \subseteq \mathbb{C}^{2} \rightarrow \mathcal{S}_{\text {reg }} \subseteq \mathbb{C}^{2}:(\xi, \eta) \mapsto\left(\xi, \mathrm{e}^{2 \pi i / n} \eta\right)
$$

In $\S 3$ we build a model $\widetilde{\mathcal{S}}_{\text {reg }}$ of the affine Riemann surface $\mathcal{S}_{\text {reg. The quadrilateral } Q \text { is }}$ holomorphically diffeomorphic to a fundamental domain $\mathcal{D}$ of the action of the covering group on $\mathcal{S}_{\text {reg. }}$ Rotating $Q$ by

$$
R: \mathbb{C} \rightarrow \mathbb{C}: z \mapsto \mathrm{e}^{2 \pi i / n} z
$$

gives a regular stellated $n$-gon $K^{*}$, which is invariant under the dihedral group $G$ generated by the mappings $R$ and $U: \mathbb{C} \rightarrow \mathbb{C}: z \mapsto \bar{z}$. We study the group theoretic properties of $K^{*}$. We show that $K^{*}$ is invariant under the reflection $S^{(j)}=R^{n_{j}} U$ in the ray $\left\{t \mathrm{e}^{2 \pi i n_{n} / n} \in \mathbb{C} \mid t \geq 0\right\}$ for $j=0,1, \infty$. To construct the model $\widetilde{\mathcal{S}}_{\text {reg }}$ of the affine Riemann surface $\mathcal{S}_{\text {reg }}$ from the regular stellated $n$-gon $K^{*}$ we follow Richens and Berry [2]. We identify two nonadjacent closed edges of $\mathrm{cl}\left(K^{*}\right)$, the closure of $K^{*}$, if one edge is obtained from the other by a reflection $S_{k}^{(j)}=R^{k} S^{(j)} R^{-k}$ for some $j=0,1, \infty$. The identification space $\left(\mathrm{cl}\left(K^{*}\right) \backslash \mathrm{O}\right)^{\sim}$, where $\mathrm{O}$ is the center of $K^{*}$, is a complex manifold except at points 
corresponding to $\mathrm{O}$ or a vertex of $\mathrm{cl}\left(K^{*}\right)$, where it has a conical singularity. The action of $G$ on $K^{*} \backslash \mathrm{O}$ induces a free and proper action on the identification space $\left(K^{*} \backslash \mathrm{O}\right)^{\sim}$, whose orbit space $\widetilde{\mathcal{S}}_{\text {reg }}$ is a complex manifold with compact closure in $\mathbb{C P}^{2}$, with genus $\frac{1}{2}\left(n+2-\left(d_{0}+d_{1}+d_{\infty}\right)\right)$. Moreover $\widetilde{\mathcal{S}}_{\text {reg }}$ is holomorphically diffeomorphic to the affine Riemann surface $\mathcal{S}_{\text {reg }}$.

In $\S 4$, we construct an affine model $\widetilde{S}_{\text {reg }}$ of the Riemann surface $\mathcal{S}_{\text {reg. }}$ In other words, we find a discrete subgroup $\mathfrak{G}$ of the 2-dimensional Euclidean group $\mathrm{E}(2)$, which acts freely and properly on $\mathbb{C} \backslash \mathbb{V}^{+}$such that after forming an identification space $\left(\mathbb{C} \backslash \mathbb{V}^{+}\right)^{\sim}$ the $\mathfrak{G}$ orbit space $\left(\mathbb{C} \backslash \mathbb{V}^{+}\right)^{\sim} / \mathfrak{G}$ is holomorphically diffeomorphic to $\mathcal{S}_{\text {reg. }}$. We now describe the group $\mathfrak{G}$. Reflect the regular stellated $n$-gon $K^{*}$ in its edges, and then in the edges of the reflected regular stellated $n$-gons, et cetera. We obtain a group $\mathcal{T}$ generated by $2 n$ translations $\tau_{k}$ so that $\tau_{1}^{\ell_{1}} \circ \cdots \circ \tau_{2 n}^{\ell_{2 n}}$ sends the center $\mathrm{O}$ of $K^{*}$ to the center of a repeatedly reflected reflected $n$-gon. The set $\mathbb{V}^{+}$is the union of the image under $\tau_{1}^{\ell_{1}} \circ \cdots \circ \tau_{2 n}^{\ell_{2 n}}$ of a vertex of $\operatorname{cl}\left(K^{*}\right)$ and its center $\mathrm{O}$ for every $\left(\ell_{1}, \ldots, \ell_{2 n}\right) \in\left(\mathbb{Z}_{\geq 0}\right)^{2 n}$. Let $\mathfrak{G}$ be the semi-direct product $G \ltimes \mathcal{T}$. The fundamental domain of the $\mathfrak{G}$ action on $\mathbb{C} \backslash \mathbb{V}^{+}$is $\operatorname{cl}\left(K^{*}\right)$ less its vertices and center. Identifying equivalent open edges of $K^{*} \backslash \mathrm{O}$ and then taking $G$ orbits, it follows that the affine model $\widetilde{S}_{\text {reg }}$ of the affine Riemann surface $\mathcal{S}_{\text {reg }}$ is the $\mathfrak{G}$ orbit space $\left(C \backslash \mathbb{V}^{+}\right)^{\sim} / \mathfrak{G}$.

In $\S 5$ we show that the mapping

$$
\delta_{Q}: \mathcal{D} \subseteq \mathcal{S}_{\text {reg }} \subseteq \mathbb{C}^{2} \rightarrow Q \subseteq \mathbb{C}:(\xi, \eta) \mapsto\left(F_{Q^{\circ}} \widehat{\pi}\right)(\xi, \eta)=z
$$

straightens the nowhere vanishing holomorphic vector field $X$ (11) on $\mathcal{S}_{\text {reg, that is, }}$ $T_{(\xi, \eta)} \delta_{Q} X(\xi, \eta)=\left.\frac{\partial}{\partial z}\right|_{z=\delta_{Q}(\xi, \eta)}$ for every $(\xi, \eta) \in \mathcal{D}$. We pull back the flat metric $\gamma=\mathrm{d} z \odot \mathrm{d} \bar{z}$

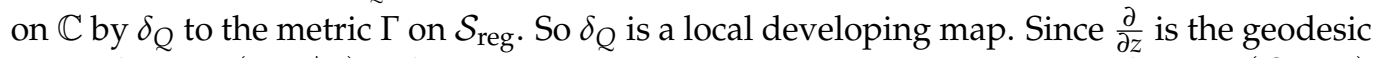
vector field on $\left(Q,\left.\gamma\right|_{Q}\right)$, it follows that $X$ is a holomorphic geodesic vector field on $\left(\mathcal{S}_{\text {reg }}, \Gamma\right)$.

In $\S 6$ we study the geometry of the developing map $\delta_{Q}$. The dihedral group $\mathcal{G}$ generated by $\mathcal{R}$ and $\mathcal{U}: \mathcal{S}_{\text {reg }} \rightarrow \mathcal{S}_{\text {reg }}:(\xi, \eta) \mapsto(\bar{\xi}, \bar{\eta})$ is a group of isometries of $\left(\mathcal{S}_{\text {reg }}, \Gamma\right)$. The group $G$ generated by $R$ and $U: \mathbb{C} \rightarrow \mathbb{C}: z \mapsto \bar{z}$ is a group of isometries of $\left(Q,\left.\gamma\right|_{Q}\right)$. Extend the holomorphic map $\delta_{Q}$ to a holomorphic map map $\delta_{K^{*}}: \mathcal{S}_{\text {reg }} \rightarrow K^{*}$ by requiring that $R^{j} \circ \delta_{K^{*}}=\delta_{Q^{\circ}} \mathcal{R}^{j}$ on $\mathcal{R}^{-j}(\mathcal{D})$. This works since $\mathcal{D}$ is a fundamental domain of the action of the covering group on $\mathcal{S}_{\text {reg, which implies }} \mathcal{S}_{\text {reg }}=\amalg_{0 \leq j \leq n} \mathcal{R}^{j}(\mathcal{D})$. Thus, the local holomorphic diffeomorphism $\delta_{K^{*}}$ intertwines the $\mathcal{G}$ action on $\left(\mathcal{S}_{\text {reg }}, \Gamma\right)$ with the $G$ action on $\left(K^{*},\left.\gamma\right|_{K^{*}}\right)$ and intertwines the local geodesic flow of the holomorphic geodesic vector field $X$ with the local geodesic flow of the holomorphic vector field $\frac{\partial}{\partial z}$.

Following Richens and Berry [2] we impose the condition: when a geodesic, starting at a point in $\operatorname{int}\left(\mathrm{cl}\left(K^{*}\right) \backslash \mathrm{O}\right)$, meets $\partial K^{*}$ it undergoes a reflection in the edge of $K^{*}$ that it meets. Such geodesics never meet a vertex of $\mathrm{cl}\left(K^{*}\right)$. Thus, this type of geodesic becomes a billiard motion in $K^{*} \backslash \mathrm{O}$, which is defined for all time. Billiard motions in polygons have been extensively studied. For a nice overview see Berger ([3], chpt. XI) and references therein. An argument shows that $\widehat{\mathcal{G}}$ invariant geodesics on $\left(\mathcal{S}_{\text {reg }}, \Gamma\right)$ correspond under the map $\delta_{K^{*} \backslash \mathrm{O}}$ to billiard motions on $\left(K^{*} \backslash \mathrm{O},\left.\gamma\right|_{\left(K^{*} \backslash \mathrm{O}\right)}\right)$.

Repeatedly reflecting a billiard motion in an edge of $K^{*} \backslash \mathrm{O}$ and suitable edges of suitable $\mathcal{T}$ translations of $K^{*} \backslash \mathrm{O}$ gives a straight line motion $\lambda$ on $\mathbb{C} \backslash \mathbb{V}^{+}$. The image of the segment of a billiard motion, where $\lambda$ intersects $K^{*} \backslash \mathrm{O}$, in the orbit space $\left(\mathbb{C} \backslash \mathbb{V}^{+}\right)^{\sim} / \mathfrak{G}=$ $\widetilde{S}_{\text {reg, }}$ is a geodesic. Here we use the flat Riemannian metric $\widehat{\gamma}$ on $\widetilde{S}_{\text {reg, }}$, which is induced by the $\mathfrak{G}$ invariant Euclidean metric $\gamma$ on $\mathbb{C} \backslash \mathbb{V}^{+}$restricted to $K^{*} \backslash$ O. Consequently, $\left(\widetilde{S}_{\text {reg }}, \widehat{\gamma}\right)$ is an affine analogue of the affine Riemann surface $\mathcal{S}_{\text {reg }}$ thought of as the orbit space of a discrete subgroup of $\operatorname{PGl}(2, \mathbb{C})$ acting on $\mathbb{C}$ with the Poincaré metric, see Weyl [4]. 


\section{A Schwarz-Christoffel Mapping}

Consider the conformal Schwarz-Christoffel mapping

$$
F_{T}: \mathbb{C}^{+}=\{\xi \in \mathbb{C} \mid \operatorname{Im} \xi \geq 0\} \rightarrow T=T_{n_{0} n_{1} n_{\infty}} \subseteq \mathbb{C}: \xi \mapsto \int_{0}^{\xi} \frac{\mathrm{d} w}{w^{1-\frac{n_{0}}{n}}(1-w)^{1-\frac{n_{1}}{n}}}=z
$$

of the upper half plane $\mathbb{C}^{+}$to the rational triangle $T=T_{n_{0} n_{1} n_{\infty}}$ with interior angles $\frac{n_{0}}{n} \pi$, $\frac{n_{1}}{n} \pi$, and $\frac{n_{\infty}}{n} \pi$, see Figure 1 . Here $n_{0}+n_{1}+n_{\infty}=n$ and $n_{j} \in \mathbb{Z}_{\geq 1}$ for $j=0,1$ and $\infty$ with $1 \leq n_{0} \leq n_{1} \leq n_{\infty}$. Because $n_{\infty}$ is greater than or equal to either $n_{0}$ or $n_{1}$, it follows that the corresponding side $O C$ is the longest side of the triangle $T=\triangle O C D$.

In the integrand of (1) we use the following choice of complex $n$th root. Suppose that $w \in \mathbb{C} \backslash\{0,1\}$. Let $w=r_{0} \mathrm{e}^{i \theta_{0}}$ and $1-w=r_{1} \mathrm{e}^{i \theta_{1}}$ where $r_{0}, r_{1} \in \mathbb{R}_{>0}$ and $\theta_{0}, \theta_{1} \in[0,2 \pi)$. For $w \in(0,1)$ on the real axis we have $\theta_{0}=\theta_{1}=0, w=r_{0}>0$, and $1-w=r_{1}>0$. So $\left(w^{n-n_{0}}(1-w)^{n-n_{1}}\right)^{1 / n}=\left(r_{0}^{n-n_{0}} r_{1}^{n-n_{1}}\right)^{1 / n}$. In general for $w \in \mathbb{C} \backslash\{0,1\}$, we have

$$
\left(w^{n-n_{0}}(1-w)^{n-n_{1}}\right)^{1 / n}=\left(r_{0}^{n-n_{0}} r_{1}^{n-n_{1}}\right)^{1 / n} \mathrm{e}^{i\left(\left(n-n_{0}\right) \theta_{0}+\left(n-n_{1}\right) \theta_{1}\right) / n} .
$$

From (1) we get

$$
F_{T}(0)=0, F_{T}(1)=C, \text { and } F_{T}(\infty)=D,
$$

where $C=\int_{0}^{1} \frac{\mathrm{d} w}{w^{1-\frac{n_{0}}{n}}(1-w)^{1-\frac{n_{1}}{n}}}$ and $D=\mathrm{e}^{\frac{n_{0}}{n} \pi i}\left(\frac{\sin \frac{n_{1}}{n} \pi}{\sin \frac{n_{\infty}}{n} \pi}\right) C$. Consequently, the bijective holomorphic mapping $F_{T}$ sends int $\left(\mathbb{C}^{+} \backslash\{0,1\}\right)$, the interior of the upper half plane less 0 and 1 , onto int $T$, the interior of the rational triangle $T=T_{n_{0} n_{1} n_{\infty}}$, and sends the boundary of $\mathbb{C}^{+} \backslash\{0,1\}$ to the edges of $\partial T$ less their end points $O, C$ and $D$, see Figure 1 . Thus, the image of $\mathbb{C}^{+} \backslash\{0,1\}$ under $F_{T}$ is $\operatorname{cl}(T) \backslash\{O, C, D\}$. Here $\operatorname{cl}(T)$ is the closure of $T$ in $\mathbb{C}$.

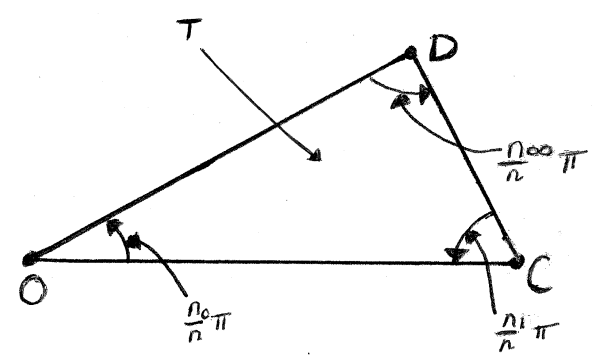

Figure 1. The rational triangle $T=T_{n_{0} n_{1} n_{\infty}}$.

Because $\left.F_{T}\right|_{[0,1]}$ is real valued, we may use the Schwarz reflection principle to extend $F_{T}$ to the holomorphic diffeomorphism

$$
F_{Q}: \mathbb{C} \backslash\{0,1\} \rightarrow Q=T \cup \bar{T} \subseteq \mathbb{C}: \xi \mapsto z= \begin{cases}F_{T}(\xi), & \text { if } \xi \in \mathbb{C}^{+} \backslash\{0,1\} \\ \overline{F_{T}(\bar{\xi}),} & \text { if } \xi \in \overline{\mathbb{C}^{+} \backslash\{0,1\}} .\end{cases}
$$

Here $Q=Q_{n_{0} n_{1} n_{\infty}}$ is a quadrilateral with internal angles $2 \pi \frac{n_{0}}{n}, \pi \frac{n_{\infty}}{n}, 2 \pi \frac{n_{1}}{n}$, and $\pi \frac{n_{\infty}}{n}$ and vertices at $O, D, C$, and $\bar{D}$, see Figure 2 . The conformal mapping $F_{Q}$ sends $\mathbb{C} \backslash\{0,1\}$ onto $\operatorname{cl}(Q) \backslash\{O, D, C, \bar{D}\}$. 


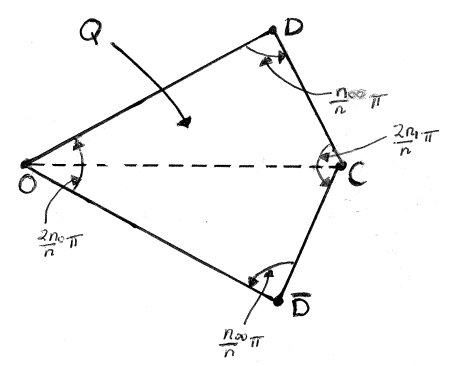

Figure 2. The rational quadrilateral $Q$.

\section{The Geometry of an Affine Riemann Surface}

Let $\xi$ and $\eta$ be coordinate functions on $\mathbb{C}^{2}$. Consider the affine Riemann surface $\mathcal{S}=\mathcal{S}_{n_{0}, n_{1}, n_{\infty}}$ in $\mathbb{C}^{2}$, associated to the holomorphic mapping $F_{Q}$, defined by

$$
g(\xi, \eta)=\eta^{n}-\xi^{n-n_{0}}(1-\xi)^{n-n_{1}}=0,
$$

see Aurell and Itzykson [1]. We determine the singular points of $\mathcal{S}$ by solving

$$
\begin{aligned}
0 & =\mathrm{d} g(\xi, \eta) \\
& =-\left(n-n_{0}\right) \xi^{n-n_{0}-1}(1-\xi)^{n-n_{1}-1}\left(1-\frac{2 n-n_{0}-n_{1}}{n-n_{0}} \xi\right) \mathrm{d} \xi+n \eta^{n-1} \mathrm{~d} \eta
\end{aligned}
$$

For $(\xi, \eta) \in \mathcal{S}$, we have $\mathrm{d} g(\xi, \eta)=0$ if and only if $(\xi, \eta)=(0,0)$ or $(1,0)$. Thus, the set $\mathcal{S}_{\text {sing }}$ of singular points of $\mathcal{S}$ is $\{(0,0),(1,0)\}$. So the affine Riemann surface $\mathcal{S}_{\text {reg }}=\mathcal{S} \backslash \mathcal{S}_{\text {sing }}$ is a complex submanifold of $\mathbb{C}^{2}$. Actually, $\mathcal{S}_{\text {reg }} \subseteq \mathbb{C}^{2} \backslash\{\eta=0\}$, for if $(\xi, \eta) \in \mathcal{S}$ and $\eta=0$, then either $\xi=0$ or $\xi=1$.

Lemma 1. Topologically $\mathcal{S}_{\text {reg }}$ is a compact Riemann surface $\overline{\mathcal{S}} \subseteq \mathbb{C P}^{2}$ of genus $g=\frac{1}{2}(n+2-$ $\left.\left(d_{0}+d_{1}+d_{\infty}\right)\right)$ less three points: $[0: 0: 1],[1: 0: 1]$, and $[0: 1: 0]$. Here $d_{j}=\operatorname{gcd}\left(n_{j}, n\right)$ for $j=0,1, \infty$.

Proof. Consider the (projective) Riemann surface $\overline{\mathcal{S}} \subseteq \mathbb{C P}^{2}$ specified by the condition $[\xi: \eta: \zeta] \in \overline{\mathcal{S}}$ if and only if

$$
G(\xi, \eta, \zeta)=\zeta^{n-n_{0}-n_{1}} \eta^{n}-\xi^{n-n_{0}}(\zeta-\xi)^{n-n_{1}}=0 .
$$

Thinking of $G$ as a polynomial in $\eta$ with coefficients which are polynomials in $\xi$ and $\zeta$, we may view $\overline{\mathcal{S}}$ as the branched covering

$$
\bar{\pi}: \overline{\mathcal{S}} \subseteq \mathbb{C P}^{2} \rightarrow \mathbb{C P}:[\xi: \eta: \zeta] \mapsto[\xi: \zeta] .
$$

When $\zeta=1$ we get the affine branched covering

$$
\pi=\bar{\pi} \mid \mathcal{S}: \mathcal{S}=\overline{\mathcal{S}} \cap\{\zeta=1\} \subseteq \mathbb{C}^{2} \rightarrow \mathbb{C}=\mathbb{C P} \cap\{\zeta=1\}:(\xi, \eta) \mapsto \zeta .
$$

From (3) it follows that $\eta=\omega_{k}\left(\xi^{n-n_{0}}(1-\xi)^{n-n_{1}}\right)^{1 / n}$, where $\omega_{k}$ for $k=0,1, \ldots, n-1$ is an $n$th root of unity with and ()$^{1 / n}$ is the complex $n$th root used in the definition of the mapping $F_{T}(1)$. Thus, the branched covering mapping $\bar{\pi}(6)$ has $n$ "sheets" except at its branch points. Since

$$
\eta=\xi^{1-\frac{n_{0}}{n}}(1-\xi)^{1-\frac{n_{1}}{n}}=\xi^{1-\frac{n_{0}}{n}}\left(1-\left(1-\frac{n_{1}}{n}\right) \xi+\cdots\right)
$$

and

$$
\eta=(1-\xi)^{1-\frac{n_{1}}{n}}(1-(1-\xi))^{1-\frac{n_{0}}{n}}
$$




$$
=(1-\xi)^{1-\frac{n_{1}}{n}}\left(1-\left(1-\frac{n_{0}}{n}\right)(1-\xi)+\cdots\right),
$$

it follows that $\xi=0$ and $\xi=1$ are branch points of the mapping $\bar{\pi}$ of degree $\frac{n}{d_{0}}$ and $\frac{n}{d_{1}}$, since $d_{j}=\operatorname{gcd}\left(n, n_{j}\right)=\operatorname{gcd}\left(n-n_{j}, n_{j}\right)$ for $j=0,1$, see McKean and Moll ([5], p. 39). Because

$$
\begin{aligned}
\eta & =\left(\frac{1}{\xi}\right)^{-\left(1-\frac{n_{0}}{n}\right)}\left(1-\frac{1}{\frac{1}{\xi}}\right)^{1-\frac{n_{1}}{n}}=(-1)^{1-\frac{n_{1}}{n}} \xi^{2-\frac{n_{0}+n_{1}}{n}}\left(1-\frac{1}{\xi}\right)^{1-\frac{n_{1}}{n}} \\
& =(-1)^{1-\frac{n_{1}}{n}} \xi^{1+\frac{n_{\infty}}{n}}\left(1-\left(1-\frac{n_{1}}{n}\right) \frac{1}{\xi}+\cdots\right)
\end{aligned}
$$

$\infty$ is a branch point of the mapping $\bar{\pi}$ of degree $\frac{n}{d_{\infty}}$, where $d_{\infty}=\operatorname{gcd}\left(n, n_{\infty}\right)$. Hence the ramification index of $0,1, \infty$ is $d_{0}\left(\frac{n}{d_{0}}-1\right)=n-d_{0}, n-d_{1}$, and $n-d_{\infty}$, respectively. Thus, the map $\bar{\pi}$ has $d_{0}$ fewer sheets at $0, d_{1}$ fewer at 1 , and $d_{\infty}$ fewer at $\infty$ than an $n$-fold covering of $\mathbb{C P}$. Thus, the total ramification index $r$ of the mapping $\bar{\pi}$ is $r=\left(n-d_{0}\right)+\left(n-d_{1}\right)+\left(n-d_{\infty}\right)$. By the Riemann-Hurwitz formula, the genus $g$ of $\overline{\mathcal{S}}$ is $r=2 n+2 g-2$. In other words,

$$
g=\frac{1}{2}\left(n+2-\left(d_{0}+d_{1}+d_{\infty}\right)\right)
$$

Consequently, the affine Riemann surface $\mathcal{S}$ is the compact connected surface $\overline{\mathcal{S}}$ less the point at $\infty$, namely, $\mathcal{S}=\overline{\mathcal{S}} \backslash\{[0: 1: 0]\}$. So $\mathcal{S}_{\text {reg }}$ is the compact connected surface $\overline{\mathcal{S}}$ less three points: $[0: 0: 1],[1: 0: 1]$, and $[0: 1: 0]$.

Examples of $\overline{\mathcal{S}}=\overline{\mathcal{S}}_{n_{0}, n_{1}, n_{\infty}}$

1. $n_{0}=1, n_{0}=1, n_{\infty}=4 ; n=6$. So $d_{0}=1, d_{1}=1, d_{\infty}=2$. Hence

$$
2 g=8-4=4 \text {. So } g=2 \text {. }
$$

2. $n_{0}=2, n_{1}=2, n_{\infty}=3 ; n=7$. So $d_{0}=d_{1}=d_{\infty}=1$. Hence

\begin{tabular}{|c|c|c|c|}
\hline$g$ & $n_{0}, n_{1}, n_{\infty} ; n$ & $g$ & $n_{0}, n_{1}, n_{\infty} ; n$ \\
\hline 1 & $1,1,1 ; 3$ & 3 & $2,2,3 ; 7$ \\
\hline 1 & $1,1,2 ; 4$ & 3 & $1,3,3$ \\
\hline 1 & $1,2,3 ; 6$ & 3 & $1,1,5$ \\
\hline 2 & $1,2,2 ; 5$ & 3 & $2,3,3 ; 8$ \\
\hline 2 & $1,1,3 ; 5$ & 3 & $1,2,5 ; 8$ \\
\hline 2 & $1,1,4 ; 6$ & 3 & $1,1,6$ \\
\hline 2 & $1,3,4 ; 8$ & 3 & $2,3,4 ; 9$ \\
\hline 2 & $2,3,5 ; 10$ & 3 & $1,3,5 ; 9$ \\
\hline \multirow[t]{6}{*}{2} & $1,4,5 ; 10$ & 3 & $1,2,6$ \\
\hline & & 3 & $3,4,5 ; 12$ \\
\hline & & 3 & $1,5,6 ; 12$ \\
\hline & & 3 & $1,3,8 ; 12$ \\
\hline & & 3 & $2,5,7 ; 14$ \\
\hline & & 3 & $1,6,7 ; 14$ \\
\hline
\end{tabular}

$2 g=9-3=6$. So $g=3$.

Table 1 below lists all the partitions $\left\{n_{1}, n_{0}, n_{\infty}\right\}$ of $n$, which give a low genus Riemann surface $\overline{\mathcal{S}}=\overline{\mathcal{S}}_{n_{0}, n_{1}, n_{\infty}}$

Table 1. Based on the table in Aurell and Itzykson ([1], p. 193).

Corollary 1. If $n$ is an odd prime number and $\left\{n_{1}, n_{0}, n_{\infty}\right\}$ is a partition of $n$ into three parts, then the genus of $\overline{\mathcal{S}}$ is $\frac{1}{2}(n-1)$.

Proof. Because $n$ is prime, we get $d_{0}=d_{1}=d_{\infty}=1$. Using the formula $g=\frac{1}{2}(n+2-$ $\left.\left(d_{0}+d_{1}+d_{\infty}\right)\right)$ we obtain $g=\frac{1}{2}(n-1)$. 
Corollary 2. The singular points of the Riemann surface $\overline{\mathcal{S}}$ are $[0: 0: 1],[1: 0: 1]$, and if $n_{\infty}>1$ then also $[0: 1: 0]$.

Proof. A point $[\xi: \eta: \zeta] \in \overline{\mathcal{S}}_{\text {sing }}$ if and only if $[\xi: \eta: \zeta] \in \overline{\mathcal{S}}$, that is,

$$
0=G(\xi, \eta, \zeta)=\zeta^{n-\left(n_{0}+n_{1}\right)} \eta^{n}-\xi^{n-n_{0}}(\zeta-\xi)^{n-n_{1}}
$$

and

$$
\begin{aligned}
&(0,0,0)= D G(\xi, \eta, \zeta) \\
&=\left(-\xi^{n-n_{0}-1}(\zeta-\xi)^{n-n_{1}-1}\left(\left(n-n_{0}\right)(\zeta-\xi)-\left(n-n_{1}\right) \xi\right)\right. \\
& \quad n \eta^{n-1} \zeta^{n-\left(n_{0}+n_{1}\right)},\left(n-\left(n_{0}+n_{1}\right)\right) \eta^{n} \zeta^{n-n_{0}-n_{1}-1} \\
&\left.\quad-\left(n-n_{1}\right) \xi^{n-n_{0}}(\zeta-\xi)^{n-n_{1}-1}\right)
\end{aligned}
$$

We need only check the points $[0: 0: 1],[1: 0: 1]$ and $[0: 1: 0]$. Since the first two points are singular points of $\mathcal{S}=\overline{\mathcal{S}} \backslash\{[0: 1: 0]\}$, they are singular points of $\overline{\mathcal{S}}$. Thus, we need to see if $[0: 1: 0]$ is a singular point of $\mathcal{\mathcal { S }}$. Substituting $(0,1,0)$ into the right hand side of $(10 \mathrm{~b})$ we get $\left\{\begin{array}{l}(0,0,1) \text {, if } n_{\infty}=n-\left(n_{0}+n_{1}\right)=1 \\ (0,0,0), \text { if } n_{\infty}>1 .\end{array}\right.$ Thus, $[0: 1: 0]$ is a singular point of $\overline{\mathcal{S}}$ only if $n_{\infty}>1$.

Lemma 2. The mapping

$$
\widehat{\pi}=\pi \mid \mathcal{S}_{\text {reg }}: \mathcal{S}_{\text {reg }} \subseteq \mathbb{C}^{2} \rightarrow \mathbb{C} \backslash\{0,1\}:(\xi, \eta) \mapsto \xi
$$

is a surjective holomorphic local diffeomorphism.

Proof. Let $(\xi, \eta) \in \mathcal{S}_{\text {reg }}$ and let

$$
X(\xi, \eta)=\eta \frac{\partial}{\partial \xi}+\frac{n-n_{0}}{n} \frac{\xi^{n-n_{0}-1}(1-\xi)^{n-n_{1}-1}\left(1-\frac{2 n-n_{0}-n_{1}}{n-n_{0}} \xi\right)}{\eta^{n-2}} \frac{\partial}{\partial \eta} .
$$

By hypothesis $(\xi, \eta) \in \mathcal{S}_{\text {reg }}$ implies that $\eta \neq 0$. The vector $X(\xi, \eta)$ is defined and is nonzero. From $(X\lrcorner \mathrm{d} g)(\xi, \eta)=0$ and $T_{(\xi, \eta)} \mathcal{S}_{\text {reg }}=\operatorname{ker} \mathrm{d} g(\xi, \eta)$, it follows that $X(\xi, \eta) \in$ $T_{(\xi, \eta)} \mathcal{S}_{\text {reg. }}$. Using the definition of $X(\xi, \eta)(12)$ and the definition of the mapping $\pi(7)$, we see that the tangent of the mapping $\widehat{\pi}(11)$ at $(\xi, \eta) \in \mathcal{S}_{\text {reg }}$ is given by

$$
T_{(\xi, \eta)} \widehat{\pi}: T_{(\xi, \eta)} \mathcal{S}_{\text {reg }} \rightarrow T_{\xi}(\mathbb{C} \backslash\{0,1\})=\mathbb{C}: X(\xi, \eta) \mapsto \eta \frac{\partial}{\partial \xi} .
$$

Since $X(\xi, \eta)$ and $\eta \frac{\partial}{\partial \xi}$ are nonzero vectors, they form a complex basis for $T_{(\xi, \eta)} \mathcal{S}_{\text {reg }}$ and $T_{\xi}(\mathbb{C} \backslash\{0,1\})$, respectively. Thus, the complex linear mapping $T_{(\xi, \eta)} \widehat{\pi}$ is an isomorphism. Hence $\widehat{\pi}$ is a local holomorphic diffeomorphism.

Corollary 3. $\widehat{\pi}(11)$ is a surjective holomorphic $n$ to 1 covering map.

Proof. We only need to show that $\widehat{\pi}$ is a covering map. First we note that every fiber of $\widehat{\pi}$ is a finite set with $n$ elements, since for each fixed $\xi \in \mathbb{C} \backslash\{0,1\}$ we have $\widehat{\pi}^{-1}(\xi)=\{(\xi, \eta) \in$ $\left.\mathcal{S}_{\text {reg }} \mid \eta=\omega_{k}\left(\xi^{n-n_{0}}(1-\xi)^{n-n_{1}}\right)^{1 / n}\right\}$. Here $\omega_{k}$ for $k=0,1, \ldots, n-1$, is an $n^{\text {th }}$ root of 1 and ()$^{1 / n}$ is the complex $n^{\text {th }}$ root used in the definition of the Schwarz-Christoffel map $F_{Q}$ (2). Hence the map $\widehat{\pi}$ is a proper surjective holomorphic submersion, because each fiber is compact. Thus, the mapping $\hat{\pi}$ is a presentation of a locally trivial fiber bundle with fiber consisting of $n$ distinct points. In other words, the map $\widehat{\pi}$ is a $n$ to 1 covering mapping. 
Consider the group $\widehat{\mathcal{G}}$ of linear transformations of $\mathbb{C}^{2}$ generated by

$$
\mathcal{R}: \mathbb{C}^{2} \rightarrow \mathbb{C}^{2}:(\xi, \eta) \mapsto\left(\xi, \mathrm{e}^{2 \pi i / n} \eta\right) .
$$

Clearly $\mathcal{R}^{n}=\mathrm{id}_{\mathbb{C}^{2}}=e$, the identity element of $\widehat{\mathcal{G}}$ and $\widehat{\mathcal{G}}=\left\{e, \mathcal{R}, \ldots, \mathcal{R}^{n-1}\right\}$. For each $(\xi, \eta) \in \mathcal{S}$ we have

$$
\left(\mathrm{e}^{2 \pi i / n} \eta\right)^{n}-\xi^{n-n_{0}}(1-\xi)^{n-n_{1}}=\eta^{n}-\xi^{n-n_{0}}(1-\xi)^{n-n_{1}}=0 .
$$

So $\mathcal{R}(\xi, \eta) \in \mathcal{S}$. Thus, we have an action of $\widehat{\mathcal{G}}$ on the affine Riemann surface $\mathcal{S}$ given by

$$
\Phi: \widehat{\mathcal{G}} \times \mathcal{S} \rightarrow \mathcal{S}:(g,(\xi, \eta)) \mapsto g(\xi, \eta) .
$$

Since $\widehat{\mathcal{G}}$ is finite, and hence is compact, the action $\Phi$ is proper. For every $g \in \widehat{\mathcal{G}}$ we have $\Phi_{g}(0,0)=(0,0)$ and $\Phi_{g}(1,0)=(1,0)$. So $\Phi_{g}$ maps $\mathcal{S}_{\text {reg }}$ into itself. At $(\xi, \eta) \in \mathcal{S}_{\text {reg }}$ the isotropy group $\widehat{\mathcal{G}}_{(\xi, \eta)}$ is $\{e\}$, that is, the $\widehat{\mathcal{G}}$-action $\Phi$ on $\mathcal{S}_{\text {reg }}$ is free. Thus, the orbit space $\mathcal{S}_{\text {reg }} / \widehat{\mathcal{G}}$ is a complex manifold.

Corollary 4. Consider the holomorphic mapping

$$
\rho: \mathcal{S}_{\text {reg }} \subseteq \mathbb{C}^{2} \rightarrow \mathcal{S}_{\text {reg }} / \widehat{\mathcal{G}} \subseteq \mathbb{C}^{2}:(\xi, \eta) \mapsto[(\xi, \eta)],
$$

where $[(\xi, \eta)]$ is the $\widehat{\mathcal{G}}$-orbit $\left\{\Phi_{g}(\xi, \eta) \in \mathcal{S}_{\text {reg }} \mid g \in \widehat{\mathcal{G}}\right\}$ of $(\xi, \eta)$ in $\mathcal{S}_{\text {reg. }}$. The $\widehat{\mathcal{G}}$ principal bundle presented by the mapping $\rho$ is isomorphic to the bundle presented by the mapping $\widehat{\pi}$ (11).

Proof. We use invariant theory to determine the orbit space $\mathcal{S} / \widehat{\mathcal{G}}$. The algebra of polynomials on $\mathbb{C}^{2}$, which are invariant under the $\widehat{\mathcal{G}}$-action $\Phi$, is generated by $\pi_{1}=\xi$ and $\pi_{2}=\eta^{n}$. Since $(\xi, \eta) \in \mathcal{S}$, these polynomials are subject to the relation

$$
\pi_{2}-\pi_{1}^{n-n_{0}}\left(1-\pi_{1}\right)^{n-n_{1}}=\eta^{n}-\xi^{n-n_{0}}(1-\xi)^{n-n_{1}}=0 .
$$

Equation (15) defines the orbit space $\mathcal{S} / \widehat{\mathcal{G}}$ as a complex subvariety of $\mathbb{C}^{2}$. This subvariety is homeomorphic to $\mathbb{C}$, because it is the graph of the function $\pi_{1} \mapsto \pi_{1}^{n-n_{0}}\left(1-\pi_{1}\right)^{n-n_{1}}$. Consequently, the orbit space $\mathcal{S}_{\text {reg }} / \widehat{\mathcal{G}}$ is holomorphically diffeomorphic to $\mathbb{C} \backslash\{0,1\}$.

It remains to show that $\widehat{\mathcal{G}}$ is the group of covering transformations of the bundle presented by the mapping $\widehat{\pi}$ (11). For each $\xi \in \mathbb{C} \backslash\{0,1\}$ look at the fiber $\hat{\pi}^{-1}(\xi)$. If $(\xi, \eta) \in$ $\widehat{\pi}^{-1}(\xi)$, then $\mathcal{R}^{ \pm 1}(\xi, \eta)=\left(\xi, \mathrm{e}^{ \pm 2 \pi i / n} \eta\right) \in \mathcal{S}_{\text {reg }}$, since $\left(\xi, \mathrm{e}^{ \pm 2 \pi i / n} \eta\right) \neq(0,0)$ or $(1,0)$ and $\left(\xi, \mathrm{e}^{ \pm 2 \pi i / n} \eta\right) \in \mathcal{S}$. Thus, $\Phi_{\mathcal{R}^{ \pm 1}}\left(\hat{\pi}^{-1}(\xi)\right) \subseteq \widehat{\pi}^{-1}(\xi)$. So $\widehat{\pi}^{-1}(\xi) \subseteq \Phi_{\mathcal{R}}\left(\hat{\pi}^{-1}(\xi)\right) \subseteq \widehat{\pi}^{-1}(\xi)$. Hence $\Phi_{\mathcal{R}}\left(\hat{\pi}^{-1}(\xi)\right)=\widehat{\pi}^{-1}(\xi)$. Thus, $\Phi_{\mathcal{R}}$ is a covering transformation for the bundle presented by the mapping $\widehat{\pi}$. So $\widehat{\mathcal{G}}$ is a subgroup of the group of covering transformations. These groups are equal because $\widehat{\mathcal{G}}$ acts transitively on each fiber of the mapping $\widehat{\pi}$.

\section{Another Model for $\mathcal{S}_{\text {reg }}$}

We construct another model $\widetilde{\mathcal{S}}_{\text {reg }}$ for the smooth part $\mathcal{S}_{\text {reg }}$ of the affine Riemann surface $\mathcal{S}$ (3) as follows. Let $\mathcal{D} \subseteq \mathcal{S}_{\text {reg }}$ be a fundamental domain for the $\widehat{\mathcal{G}}$ action $\Phi$ (14) on $\mathcal{S}_{\text {reg. }}$. So $(\xi, \eta) \in \mathcal{D}$ if and only if for $\xi \in \mathbb{C} \backslash\{0,1\}$ we have $\eta=\left(\xi^{n-n_{0}}(1-\xi)^{n-n_{1}}\right)^{1 / n}$. Here ()$^{1 / n}$ is the $n^{\text {th }}$ root used in the definition of the mapping $F_{Q}(2)$. The domain $\mathcal{D}$ is a connected

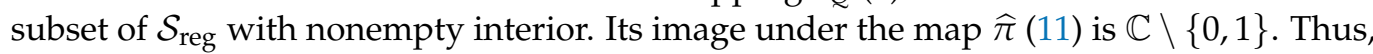
$\mathcal{D}$ is one "sheet" of the covering map $\widehat{\pi}$. So $\left.\widehat{\pi}\right|_{\mathcal{D}}$ is one to one.

Using the extended Schwarz-Christoffel mapping $F_{Q}(2)$, we give a more geometric description of the fundamental domain $\mathcal{D}$. Consider the mapping

$$
\delta: \mathcal{D} \subseteq \mathcal{S}_{\text {reg }} \rightarrow Q \subseteq \mathbb{C}:(\xi, \eta) \mapsto F_{Q}(\widehat{\pi}(\xi, \eta)),
$$


where the map $\hat{\pi}$ is given by Equation (11). The map $\delta$ is a holomorphic diffeomorphism of int $\mathcal{D}$ onto int $Q$, which sends $\partial \mathcal{D}$ homeomorphically onto $\partial Q$. Look at $\operatorname{cl}(Q)$, which is a closed quadrilateral with vertices $O, D, C$, and $\bar{D}$. The set $\delta(\mathcal{D})$ contains the open edges $O D, D C$, and $C \bar{D}$ but not the open edge $O \bar{D}$ of $\mathrm{cl}(Q)$, see Figure 3 above.

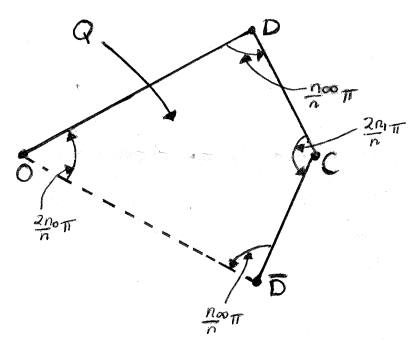

Figure 3. The image $Q$ of the fundamental domain $\mathcal{D}$ under the mapping $\delta$. The open edges $O D, C \bar{D}$, and $C D$ of the quadrilateral are included; while the open edge $O \bar{D}$ is excluded.

Let $K^{*}=K_{n_{0}, n_{1}, n_{\infty}}^{*}=\amalg_{0 \leq j \leq n-1} R^{j}(\delta(\mathcal{D}))$ be the region in $\mathbb{C}$ formed by repeatedly rotating $Q=\delta(\mathcal{D})$ through an angle $2 \pi / n$. Here $R$ is the rotation $\mathbb{C} \rightarrow \mathbb{C}: z \mapsto \mathrm{e}^{2 \pi i / n} z$. We say that the quadrilateral $Q=Q_{2 n_{0}, n_{\infty}, 2 n_{1}, n_{\infty}}$ forms $K^{*}$, see Figure 4 above.

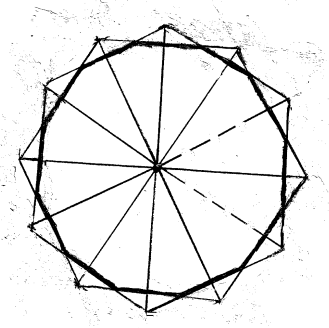

Figure 4. The regular duodecagon $K$ and the stellated regular duodecagon $K^{*}=K_{4,4,4}^{*}$ formed by rotating the quadrilateral $Q_{4,4,4}$ through an angle $2 \pi / 12$ around the origin.

Theorem 1. The connected set $K^{*}$ is a regular stellated $n$-gon with its $2 n$ vertices omitted, which is formed from the quadrilateral $Q^{\prime}=O D^{\prime} C \overline{D^{\prime}}$, see Figure 5 .

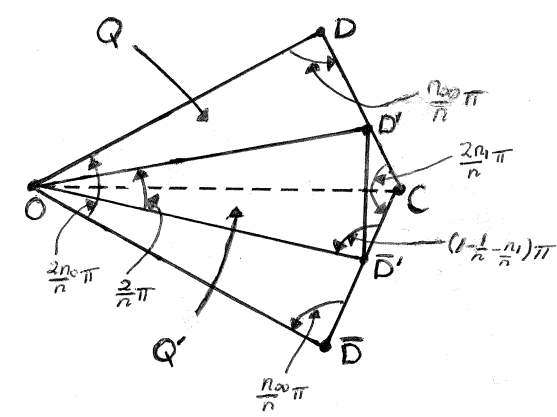

Figure 5. The dart in the figure is the quadrilateral $Q^{\prime}=O D^{\prime} C \overline{D^{\prime}}$, which is the union of the triangles $T=\triangle O D^{\prime} C$ and the triangle $\overline{T^{\prime}}$.

Proof. By construction the quadrilateral $Q^{\prime}=O D^{\prime} C \overline{D^{\prime}}$ is contained in the quadrilateral $Q=O D C \bar{D}$. Note that $Q \subseteq \bigcup_{j=\left[-\frac{n_{1}+1}{2}\right]}^{\left[\frac{n_{1}+1}{2}\right]} R^{j}\left(Q^{\prime}\right)$. Thus,

$$
K^{*}=\bigcup_{j=0}^{n} R^{j}(Q) \subseteq \bigcup_{j=0}^{n} R^{j}\left(Q^{\prime}\right) \subseteq \bigcup_{j=0}^{n} R^{j}(Q)=K^{*} .
$$


So $K^{*}=\bigcup_{j=0}^{n} R^{j}\left(Q^{\prime}\right)$. Thus, $K^{*}$ is the regular stellated $n$-gon less its vertices, one of whose open sides is the diagonal $D^{\prime} \overline{D^{\prime}}$ of $Q^{\prime}$.

We would like to extend the mapping $\delta(16)$ to a mapping of $\mathcal{S}_{\text {reg }}$ onto $K^{*}$. Let

$$
\delta_{\Phi_{\mathcal{R}^{j}}(\mathcal{D})}: \Phi_{\mathcal{R}^{j}}(\mathcal{D}) \subseteq \mathcal{S}_{\text {reg }} \rightarrow R^{j}(\delta(\mathcal{D})) \subseteq K^{*}:(\xi, \eta) \mapsto R^{j} \delta\left(\Phi_{\mathcal{R}^{-j}}(\xi, \eta)\right),
$$

where $\Phi$ is the $\widehat{\mathcal{G}}$ action defined in Equation (14). So we have a mapping

$$
\delta_{K^{*}}: \mathcal{S}_{\text {reg }} \subseteq \mathbb{C}^{2} \rightarrow K^{*} \subseteq \mathbb{C}
$$

defined by $\left.\left(\delta_{K^{*}}\right)\right|_{\Phi_{\mathcal{R}^{j}}(\mathcal{D})}=\left.\delta\right|_{\Phi_{\mathcal{R}^{j}}(\mathcal{D})}$. The mapping $\delta_{K^{*}}$ is defined on $\mathcal{S}_{\text {reg }}$, because $\mathcal{S}_{\text {reg }}=$ $\amalg_{0 \leq j \leq n-1} \Phi_{\mathcal{R}^{j}}(\mathcal{D})$, since $\mathcal{D}$ is a fundamental domain for the $\widehat{\mathcal{G}}$-action $\Phi(14)$ on $\mathcal{S}_{\text {reg. }}$. Because $K^{*}=\amalg_{0 \leq j \leq n-1} R^{j}(\delta(\mathcal{D}))$, the mapping $\delta_{K^{*}}$ is surjective. Hence $\delta_{K^{*}}$ is holomorphic, since it is continuous on $\mathcal{S}_{\text {reg }}$ and is holomorphic on the dense open subset $\amalg_{0 \leq j \leq n-1} \mathcal{R}^{j}(\operatorname{int} \mathcal{D})$ of $\mathcal{S}_{\text {reg. }}$ Let $U: \mathbb{C} \rightarrow \mathbb{C}: z \mapsto \bar{z}$ and let $G$ be the group generated by the rotation $R$ and the reflection $U$ subject to the relations $R^{n}=U^{2}=e$ and $R U=U R^{-1}$. Shorthand $G=$ $\left\langle U, R \mid U^{2}=e=R^{n} \& R U=U R^{-1}\right\rangle$. Then $G=\left\{e ; R^{p} U^{\ell}, \ell=0,1 \& p=0,1, \ldots, n-1\right\}$. The group $G$ is the dihedral group $D_{2 n}$. The closure $\operatorname{cl}\left(K^{*}\right)$ of $K^{*}=\amalg_{0 \leq j \leq n-1} R^{j}(Q)$ in $\mathbb{C}$ is invariant under $\widehat{G}$, the subgroup of $G$ generated by the rotation $R$. Because the quadrilateral $Q$ is invariant under the reflection $U: z \mapsto \bar{z}$, and $U R^{j}=R^{-j} U$, it follows that $\operatorname{cl}\left(K^{*}\right)$ is invariant under the reflection $U$. $\operatorname{So~} \mathrm{cl}\left(K^{*}\right)$ is invariant under the group $G$.

We now look at some group theoretic properties of $K^{*}$.

Lemma 3. If $F$ is a closed edge of the polygon $\mathrm{cl}\left(K^{*}\right)$ and $\left.g\right|_{F}=\left.\mathrm{id}\right|_{F}$ for some $g \in G$, then $g=e$.

Proof. Suppose that $g \neq e$. Then $g=R^{p} U^{\ell}$ for some $\ell \in\{0,1\}$ and some $p \in\{0,1, \ldots$, $n-1\}$. Let $g=R^{p} U$ and suppose that $F$ is an edge of $\operatorname{cl}\left(K^{*}\right)$ such that $\operatorname{int}(F) \cap \mathbb{R} \neq \varnothing$, where $\mathbb{R}=\{\operatorname{Re} z \mid z \in \mathbb{C}\}$. Then $U(F)=F$, but $\left.U\right|_{F} \neq \mathrm{id}_{F}$. So $\left.g\right|_{F}=\left.R^{p} U\right|_{F} \neq \operatorname{id}_{F}$. Now suppose that $\operatorname{int}(F) \cap \mathbb{R}=\varnothing$. Then $U(F) \neq F$. So $\left.U\right|_{F} \neq \operatorname{id}_{F}$. Hence $\left.g\right|_{F} \neq \operatorname{id}_{F}$. Finally, suppose that $g=R^{p}$ with $p \neq 0$. Then $g(F) \neq F$. So $\left.g\right|_{F} \neq\left.\mathrm{id}\right|_{F}$.

Lemma 4. For $j=0,1, \infty$ put $S^{(j)}=R^{n_{j}} U$. Then $S^{(j)}$ is a reflection in the closed ray $\ell^{j}=$ $\left\{t \mathrm{e}^{i \pi n_{j} / n} \in \mathbb{C} \mid t \in O D\right\}$. The ray $\ell^{0}$ is the closure of the side $O D$ of the quadrilateral $Q=O D C \bar{D}$ in Figure 5.

Proof. $S^{(j)}$ fixes every point on the closed ray $\ell^{j}$, because

$$
S^{(j)}\left(\left\{t \mathrm{e}^{i \pi n_{j} / n} \mid t \in O D\right\}\right)=R^{n_{j}}\left(\left\{t \mathrm{e}^{-i \pi n_{j} / n} \mid t \in O D\right\}\right)=\left\{t \mathrm{e}^{i \pi n_{j} / n} \mid t \in O D\right\} .
$$

Since $\left(S^{(j)}\right)^{2}=\left(R^{n_{j}} U\right)\left(R^{n_{j}} U\right)=R^{n_{j}}(U U) R^{-n_{j}}=e$, it follows that $S^{(j)}$ is a reflection in the closed ray $\ell^{j}$.

Corollary 5. For every $j=0,1, \infty$ and every $k \in\{0,1, \ldots, n-1\}$ let $S_{k}^{(j)}=R^{k} S^{(j)} R^{-k}$. Here $S_{n}^{(j)}=S_{0}^{(j)}=S^{(j)}$, because $R^{n}=e$. Then $S_{k}^{(j)}$ is a reflection in the closed ray $R^{k} \ell^{j}$.

Proof. This follows because $\left(S_{k}^{(j)}\right)^{2}=R^{k}\left(S^{(j)}\right)^{2} R^{-k}=e$ and $S_{k}^{(j)}$ fixes every point on the closed ray $R^{k} \ell^{j}$, for

$$
\begin{gathered}
\left.S_{k}^{(j)}\left(R^{k}\left(\left\{t \mathrm{e}^{i \pi n_{j} / n} \mid t \in O D\right\}\right)\right)=R^{k} S^{(j)}\left(\left\{t \mathrm{e}^{i \pi n_{j} / n} \mid t \in O D\right\}\right)\right) \\
=R^{k}\left(\left\{t \mathrm{e}^{i \pi n_{j} / n} \mid t \in O D\right\}\right) .
\end{gathered}
$$


Corollary 6. For every $j=0,1, \infty$, every $S_{k}^{(j)}$ with $k=0,1, \ldots, n-1$, and every $g \in G$, we have $g S_{k}^{(j)} g^{-1}=S_{r}^{(j)}$ for a unique $r \in\{0,1, \ldots, n-1\}$.

Proof. We compute. For every $k=0,1, \ldots, n-1$ we have

$$
R S_{k}^{(j)} R^{-1}=R\left(R^{k} S^{(j)} R^{-k}\right) R^{-1}=R^{(k+1)} S^{(j)} R^{-(k+1)}=S_{k+1}^{(j)}
$$

and

$$
\begin{aligned}
U S_{k}^{(j)} U^{-1} & =U\left(R^{\left(k+n_{j}\right)} U R^{-\left(k+n_{j}\right)}\right) U=R^{-\left(k+n_{j}\right)} U R^{\left(k+n_{j}\right)} \\
& =S_{-\left(k+2 n_{j}\right)}^{(j)}=S_{t}^{(j)}
\end{aligned}
$$

where $t=-\left(k+2 n_{j}\right) \bmod n$. Since $R$ and $U$ generate the group $G$, the corollary follows.

Corollary 7. For $j=0,1, \infty$ let $G^{j}$ be the group generated by the reflections $S_{k}^{(j)}$ for $k=$ $0,1, \ldots, n-1$. Then $G^{j}$ is a normal subgroup of $G$.

Proof. Clearly $G^{j}$ is a subgroup of $G$. From Equations (18) and (19) it follows that $g S_{k}^{(j)} g^{-1} \in G^{j}$ for every $g \in G$ and every $k=0,1 \ldots, n-1$, since $G$ is generated by $R$ and $U$. However, $G^{j}$ is generated by the reflections $S_{k}^{(j)}$ for $k=0,1, \ldots, n-1$, that is, every $g^{\prime} \in G^{j}$ may be written as $S_{i_{1}}^{(j)} \ldots S_{i_{p}}^{(j)}$, where for $\ell \in\{1, \ldots p\}$ we have $i_{\ell} \in\{0,1, \ldots, n-1\}$. So $g g^{\prime} g^{-1}=g\left(S_{i_{1}}^{(j)} \cdots S_{i_{p}}^{(j)}\right) g^{-1}=\left(g S_{i_{1}}^{(j)} g^{-1}\right) \cdots\left(g S_{i_{p}}^{(j)} g^{-1}\right) \in G^{j}$ for every $g \in G$, that is, $G^{j}$ is a normal subgroup of $G$.

As a first step in constructing the model $\widetilde{\mathcal{S}}_{\text {reg }}$ of $\mathcal{S}_{\text {reg }}$ from the regular stellated $n$-gon $K^{*}$ we look at certain pairs of edges of $\mathrm{cl}\left(K^{*}\right)$. For each $j=0,1, \infty$ we say two distinct closed edges $E$ and $E^{\prime}$ of $\mathrm{cl}\left(K^{*}\right)$ are adjacent if and only if they intersect at a vertex of $\operatorname{cl}\left(K^{*}\right)$. For $j=0,1, \infty$ let $\mathcal{E}^{j}$ be the set of unordered pairs of equivalent closed edges $E$ and $E^{\prime}$ of $\mathrm{cl}\left(K^{*}\right)$, that is, the edges $E$ and $E^{\prime}$ are not adjacent and $E^{\prime}=S_{m}^{(j)}(E)$ for some generator $S_{m}^{(j)}$ of $G^{j}$. Recall that for $x$ and $y$ in some set, the unordered pair $[x, y]$ is precisely one of the ordered pairs $(x, y)$ or $(y, x)$. Note that $\bigcup_{j=0,1, \infty} \mathcal{E}^{j}$ is the set of all unordered pairs of nonadjacent edges of $\mathrm{cl}\left(K^{*}\right)$. Geometrically, two nonadjacent closed edges $E^{\prime}$ and $E$ of $\mathrm{cl}\left(K^{*}\right)$ are equivalent if and only if $E^{\prime}$ is obtained from $E$ by reflection in the line $R^{m} \ell^{j}$ for some $m \in\{0,1, \ldots, n-1\}$ and some $j=0,1, \infty$. In Figure 6 , where $K^{*},=K_{1,1,4^{\prime}}^{*}$ parallel edges of $K^{*}$, which are labeled with the same letter, are $G^{0}$-equivalent. This is no coincidence.

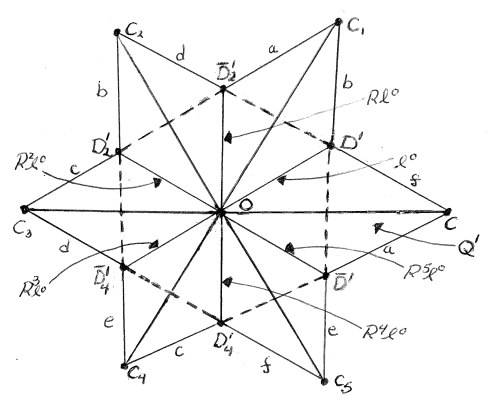

Figure 6. The triangulation $\mathcal{T}_{c l}\left(K^{*}\right)$ of the regular stellated hexagon $K^{*}$. The vertices of $\mathrm{cl}\left(K^{*}\right)$ are labeled $X_{j}=R^{j} X$ for $X=A, B, C$ and equivalent edges by $a, b, c, d, e, f$. 
Lemma 5. Let $K^{*}$ be formed from the quadrilateral $Q=T \cup \bar{T}$, where $T$ is the isosceles rational triangle $T_{n_{0} n_{0} n_{\infty}}$ less its vertices. Then nonadjacent edges of $\partial \mathrm{cl}\left(K^{*}\right)$ are $G^{0}$-equivalent if and only if they are parallel, see Figure 7.

Proof. In Figure 7, let $O A B$ be the triangle $T$ with $\angle A O B=\alpha, \angle O A B=\beta$, and $\angle A B O=\gamma$. Let $O A B A^{\prime \prime}$ be the quadrilateral formed by reflecting the triangle $O A B$ in its edge $O B$. The quadrilateral $O A B A^{\prime \prime}$ reflected it its edge $O A$ is the quadrilateral $O A B^{\prime} A^{\prime}$. Let $A C^{\prime}$ be perpendicular to $A^{\prime} B^{\prime}$ and $A C$ be perpendicular to $A^{\prime \prime} B$, see Figure 7. Then $C A C^{\prime}$ is a straight line if and only if $\angle C^{\prime} A B^{\prime}+\angle B^{\prime} A B+\angle B A C=\pi$. By construction $\angle C^{\prime} A B^{\prime}=$ $\angle B A C=\pi / 2-2 \gamma$ and $\angle B^{\prime} A B=2 \pi-2 \beta$. So

$$
\begin{aligned}
\pi & =2\left(\frac{\pi}{2}-2 \gamma\right)+2(\pi-\beta)=3 \pi-2(\beta+\gamma)-2 \gamma \\
& =3 \pi-2(\alpha+\beta+\gamma)+2(\alpha-\gamma)=\pi+2(\alpha-\gamma),
\end{aligned}
$$

if and only if $\alpha=\gamma$. Hence the edges $A^{\prime \prime} B$ and $A^{\prime} B^{\prime}$ are parallel if and only if the triangle $O A B$ is isosceles.

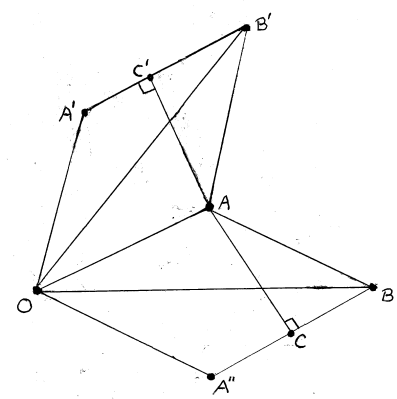

Figure 7. The geometric configuration.

Theorem 2. Let $K^{*}$ be the regular stellated $n$-gon formed from the rational quadrilateral $Q_{n_{0} n_{1} n_{\infty}}$ with $d_{j}=\operatorname{gcd}\left(n_{j}, n\right)$ for $j=0,1, \infty$. The $G$ orbit space formed by first identifiying equivalent edges of the regular stellated $n$-gon $K^{*}$ formed from $Q$ less $O$ and then acting on the identification space by the group $G$ is $\widetilde{\mathcal{S}}_{\text {reg, }}$, which is a smooth 2 -sphere with $g$ handles, where $g=\frac{1}{2}(n+2-$ $\left.\left(d_{0}+d_{1}+d_{\infty}\right)\right)$, less some points corresponding to the image of the vertices of $\mathrm{cl}\left(K^{*}\right)$.

Example 1. Before we begin proving Theorem 2 we consider the following special case. Let $K^{*}=K_{1,1,4}^{*}$ be a regular stellated hexagon formed by repeatedly rotating the quadrilateral $Q^{\prime}=$ $O D^{\prime} C \overline{D^{\prime}}$ by $R$ through an angle $2 \pi / 6$, see Figure 6 .

Let $G^{0}$ be the group generated by the reflections $S_{k}^{(0)}=R^{k} S^{(0)} R^{-k}=R^{2 k+1} U$ for $k=0,1, \ldots, 5$. Here $S^{(0)}=R U$ is the reflection which leaves the closed ray $\ell^{0}=\left\{t \mathrm{e}^{i \rho / 6} \mid t \in\right.$ $\left.O D^{\prime}\right\}$ fixed. Define an equivalence relation on $\mathrm{cl}\left(K^{*}\right)$ by saying that two points $x$ and $y$ in $\operatorname{cl}\left(K^{*}\right)$ are equivalent, $x \sim y$, if and only if 1) $x$ and $y$ lie on $\partial \operatorname{cl}\left(K^{*}\right)$ with $x$ on the closed edge $E$ and $y=S_{m}^{(0)}(x) \in S_{m}^{(0)}(E)$ for some reflection $S_{m}^{(0)} \in G^{0}$ or 2) if $x$ and $y$ lie in the interior of $\operatorname{cl}\left(K^{*}\right)$ and $x=y$. Let $\mathrm{cl}\left(K^{*}\right)^{\sim}$ be the space of equivalence classes and let

$$
\rho: \operatorname{cl}\left(K^{*}\right) \rightarrow \operatorname{cl}\left(K^{*}\right)^{\sim}: p \mapsto[p]
$$

be the identification map which sends a point $p \in \mathrm{cl}\left(K^{*}\right)$ to the equivalence class $[p]$, which contains $p$. Give $\mathrm{cl}\left(K^{*}\right)$ the topology induced from $\mathbb{C}$. Placing the quotient topology on $\mathrm{cl}\left(K^{*}\right)^{\sim}$ turns it into a connected topological manifold without boundary, whose closure is compact. Let $K^{*}$ be $c l\left(K^{*}\right)$ less its vertices. The identification space $\left(K^{*} \backslash \mathrm{O}\right) \sim=\rho\left(K^{*} \backslash \mathrm{O}\right)$ is a connected 2-dimensional smooth manifold without boundary. 
Let $G=\left\langle R, U \mid R^{6}=e=U^{2} \& R U=U R^{-1}\right\rangle$. The usual $G$-action

$$
G \times \operatorname{cl}\left(K^{*}\right) \subseteq G \times \mathbb{C} \rightarrow \operatorname{cl}\left(K^{*}\right) \subseteq \mathbb{C}:(g, z) \mapsto g(z)
$$

preserves equivalent edges of $\operatorname{cl}\left(K^{*}\right)$ and is free on $K^{*} \backslash \mathrm{O}$. Hence it induces a $G$ action on $\left(K^{*} \backslash \mathrm{O}\right)^{\sim}$, which is free and proper. Thus, its orbit map

$$
\sigma:\left(K^{*} \backslash \mathrm{O}\right)^{\sim} \rightarrow\left(K^{*} \backslash \mathrm{O}\right)^{\sim} / G=\widetilde{\mathcal{S}}_{\text {reg }}: z \mapsto z G
$$

is surjective, smooth, and open. The orbit space $\widetilde{\mathcal{S}}_{\text {reg }}=\sigma\left(\left(K^{*} \backslash \mathrm{O}\right)^{\sim}\right)$ is a connected 2-dimensional smooth manifold. The identification space $\left(K^{*} \backslash \mathrm{O}\right) \sim$ has the orientation induced from an orientation of $K^{*} \backslash \mathrm{O}$, which comes from $\mathbb{C}$. So $\widetilde{\mathcal{S}}_{\text {reg }}$ has a complex structure, since each element of $G$ is a conformal mapping of $\mathbb{C}$ into itself.

Our aim is to specify the topology of $\widetilde{\mathcal{S}}_{\text {reg. }}$. The regular stellated hexagon $K^{*} \backslash \mathrm{O}$ less the origin has a triangulation $\mathcal{T}_{K^{*} \backslash \mathrm{O}}$ made up of 12 open triangles $R^{j}\left(\triangle O C D^{\prime}\right)$ and $R^{j}\left(\triangle O C \bar{D}^{\prime}\right)$ for $j=0,1, \ldots, 5 ; 24$ open edges $R^{j}(O C), R^{j}\left(O \bar{D}^{\prime}\right), R^{j}\left(C \bar{D}^{\prime}\right)$, and $R^{j}\left(C D^{\prime}\right)$ for $j=0,1, \ldots, 5$; and 12 vertices $R^{j}\left(D^{\prime}\right)$ and $R^{j}(C)$ for $j=0,1, \ldots, 5$, see Figure 6 .

Consider the set $\mathcal{E}^{0}$ of unordered pairs of equivalent closed edges of $\mathrm{cl}\left(K^{*}\right)$, that is, $\mathcal{E}^{0}$ is the set $\left[E, S_{k}^{(0)}(E)\right]$ for $k=0,1, \ldots, 5$, where $E$ is a closed edge of $\operatorname{cl}\left(K^{*}\right)$. Table 2 lists the elements of $\mathcal{E}^{0}$. $G$ acts on $\mathcal{E}^{0}$, namely, $g \cdot\left[E, S_{k}^{(0)}(E)\right]=\left[g(E), g S_{k}^{(0)} g^{-1}(g(E))\right]$, for $g \in G$. Since $G^{0}$ is the group generated by the reflections $S_{k}^{(0)}, k=0,1, \ldots, 5$, it is a normal subgroup of $G$. Hence the action of $G$ on $\mathcal{E}^{0}$ restricts to an action of $G^{0}$ on $\mathcal{E}^{0}$ and the $G$ action permutes $G^{0}$-orbits in $\mathcal{E}^{0}$. Thus, the set of $G^{0}$-orbits in $\mathcal{E}^{0}$ is $G$-invariant.

Table 2. The set $\mathcal{E}^{0}$. Here $D_{k}^{\prime}=R^{k}\left(D^{\prime}\right)$ and $\overline{D_{k}^{\prime}}=R^{k}\left(\overline{D^{\prime}}\right)$ for $k=0,2,4$ and $C_{k}=R^{k}(C)$ for $k=\{0,1, \ldots, 5\}$, see Figure 6 .

\begin{tabular}{ll}
\hline$a=\left[\overline{D^{\prime}} C, S_{0}^{(0)}\left(\overline{D^{\prime}} C\right)=\overline{D_{2}^{\prime}} C_{1}\right]$ & $b=\left[D^{\prime} C_{1}, S_{1}^{(0)}\left(D^{\prime} C_{1}\right)=D_{2}^{\prime} C_{2}\right]$ \\
\hline$d=\left[\overline{D_{2}^{\prime}} C_{2}, S_{2}^{(0)}\left(\overline{D_{2}^{\prime}} C_{2}\right)=\overline{D_{4}^{\prime}} C_{3}\right]$ & $c=\left[D_{2}^{\prime} C_{3}, S_{3}^{(0)}\left(D_{2}^{\prime} C_{3}\right)=D_{4}^{\prime} C_{4}\right]$ \\
\hline$e=\left[\overline{D_{4}^{\prime}} C_{4}, S_{4}^{(0)}\left(\overline{D_{4}^{\prime}} C_{4}\right)=\overline{D^{\prime}} C_{5}\right]$ & $f=\left[D_{4}^{\prime} C_{5}, S_{5}^{(0)}\left(D_{4}^{\prime} C_{5}\right)=D^{\prime} C\right]$ \\
\hline
\end{tabular}

We now look at the $G^{0}$-orbits on $\mathcal{E}^{0}$. We compute the $G^{0}$-orbit of $d \in \mathcal{E}^{0}$ as follows. We have

$$
\begin{aligned}
(U R) \cdot d & \left.=\left[U R\left(\overline{D_{2}^{\prime}} C_{2}\right), U R\left(S_{2}^{(0)}\left(\overline{D_{2}^{\prime}} C_{2}\right)\right)\right]=\left[U R\left(\overline{D_{2}^{\prime}} C_{2}\right), U R\left(\overline{D_{4}^{\prime}} C_{3}\right)\right)\right] \\
& =\left[U\left(D_{2}^{\prime} C_{3}\right), U\left(D_{4}^{\prime} C_{4}\right)\right]=\left[\overline{D_{4}^{\prime}} C_{5}, \overline{D_{2}^{\prime}} C_{2}\right]=d .
\end{aligned}
$$

Since

$$
\begin{aligned}
R^{2} \cdot d & =R^{2} \cdot\left[\overline{D_{2}^{\prime}} C_{2}, S_{2}^{(0)}\left(\overline{D_{2}^{\prime}} C_{2}\right)\right]=\left[R^{2}\left(\overline{D_{2}^{\prime}} C_{2}\right), R^{2} S_{2}^{(0)} R^{-2}\left(R^{2}\left(\overline{D_{2}^{\prime}} C_{2}\right)\right)\right] \\
& =\left[\overline{D_{4}^{\prime}} C_{4}, S_{4}^{(0)}\left(\overline{D_{4}^{\prime}} C_{4}\right)\right]=\left[\overline{D_{4}^{\prime}} C_{4}, \overline{D^{\prime}} C_{5}\right]=e
\end{aligned}
$$

and

$$
\begin{aligned}
R^{4} \cdot d & =\left[R^{4}\left(\overline{D_{4}^{\prime}} C_{2}\right), R^{4} S_{2}^{(0)} R^{-4}\left(R^{4}\left(\overline{D_{2}^{\prime}} C_{2}\right)\right)\right] \\
& =\left[\overline{D^{\prime}} C, S_{6}^{(0)}\left(\overline{D^{\prime}} C\right)\right]=\left[\overline{D^{\prime}} C, S_{0}^{(0)}\left(\overline{D^{\prime}} C\right)\right]=\left[\overline{D^{\prime} C}, \overline{D_{2}^{\prime}} C_{1}\right]=a,
\end{aligned}
$$

the $G^{0}$ orbit $G^{0} \cdot d$ of $d \in \mathcal{E}^{0}$ is $\left(G^{0} /\left\langle U R \mid(U R)^{2}=e\right\rangle\right) \cdot d=H^{0} \cdot d=\{a, d, e\}$. Here $H^{0}=$ $\left\langle V=R^{2} \mid V^{3}=e\right\rangle$, since $G^{0}=\left\langle V=R^{2}, U R \mid V^{3}=e=(U R)^{2} \& V(U R)=(U R) V^{-1}\right\rangle$. Similarly, the $G^{0}$-orbit $G^{0} \cdot f$ of $f \in \mathcal{E}^{0}$ is $H^{0} \cdot f=\{b, c, f\}$. Since $G^{0} \cdot d \cup G^{0} \cdot f=\mathcal{E}^{0}$, we have found all $G^{0}$-orbits on $\mathcal{E}^{0}$. The $G$-orbit of $O C$ is $R^{j}(O C)$ for $j=0,1, \ldots, 5$, since 
$U(O C)=O C$; while the $G$-orbit of $O D^{\prime}$ is $R^{j}\left(O D^{\prime}\right), R^{j}\left(O \overline{D^{\prime}}\right)$ for $j=0,1, \ldots, 5$, since $U\left(O D^{\prime}\right)=O \overline{D^{\prime}}$.

Suppose that $B$ is an end point of the closed edge $E$ of $\operatorname{cl}\left(K^{*}\right)$. Then $E$ lies in a unique $\left[E, S_{m}^{(0)}(E)\right]$ of $\mathcal{E}^{0}$. Let $G^{0} \cdot\left[E, S_{m}^{(0)}(E)\right]$ be the $G^{0}$-orbit of $\left[E, S_{m}^{(0)}(E)\right]$. Then $g^{\prime} \cdot B$ is an end point of the closed edge $g^{\prime}(E)$ of $g^{\prime} \cdot\left[E, S_{m}^{(0)}(E)\right] \in \mathcal{E}^{0}$ for every $g^{\prime} \in G^{0}$. So $\mathcal{O}(B)=\left\{g^{\prime}\right.$. $\left.B \mid g^{\prime} \in G^{0}\right\}$ the $G^{0}$-orbit of the vertex $B$. It follows from the classification of $G^{0}$-orbits on $\mathcal{E}^{0}$ that $\mathcal{O}\left(D^{\prime}\right)=\left\{D^{\prime}, D_{2}^{\prime}, D_{4}^{\prime}\right\}$ and $\mathcal{O}\left(\overline{D^{\prime}}\right)=\left\{\overline{D^{\prime}}, \overline{D^{\prime}}{ }_{2}, \overline{D^{\prime}} 4\right\}$ are $G^{0}$-orbits of the vertices of $\mathrm{cl}\left(K^{*}\right)$, which are permuted by the action of $G$ on $\mathcal{E}^{0}$. Furthermore, $\mathcal{O}(C)=\left\{C, C_{1}, \ldots, C_{5}\right\}$ and $\mathcal{O}\left(D^{\prime} \& \overline{D^{\prime}}\right)=\left\{D^{\prime}, \overline{D^{\prime}}, D_{2}^{\prime}, \overline{D^{\prime}}{ }_{2}, D_{4}^{\prime}, \overline{D^{\prime}} 4\right\}$ are $G$-orbits of vertices of $\mathrm{cl}\left(K^{*}\right)$, which are end points of the $G$-orbit of the rays $O C$ and $O D^{\prime}$, respectively.

To determine the topology of the $G$ orbit space $\widetilde{\mathcal{S}}_{\text {reg }}$ we find a triangulation of $\widetilde{\mathcal{S}}_{\text {reg }}$. Note that the triangulation $\mathcal{T}_{K^{*} \backslash \mathrm{O}}$ of $K^{*} \backslash \mathrm{O}$, illustrated in Figure 6, is G-invariant. Its image under the identification map $\rho$ is a $G$-invariant triangulation $\mathcal{T}_{\left(K^{*} \backslash \mathrm{O}\right)^{\sim}}$ of $\left(K^{*} \backslash \mathrm{O}\right)^{\sim}$. After identification of equivalent edges, each vertex $\rho(v)$, each open edge $\rho(E)$, having $\rho(O)$ as an end point, or each open edge $\rho\left(\left[F, F^{\prime}\right]\right)$, where $\left[F, F^{\prime}\right]$ is a pair of equivalent edges of $\mathrm{cl}\left(K^{*}\right)$, and each open triangle $\rho(T)$ in $\mathcal{T}_{\left(K^{*} \backslash \mathrm{O}\right)} \sim$ lies in a unique $G$ orbit. It follows that $\sigma(\rho(v)), \sigma(\rho(E))$ or $\sigma\left(\rho\left(\left[F, F^{\prime}\right]\right)\right)$, and $\sigma(\rho(T))$ is a vertex, an open edge, and an open triangle, respectively, of a triangulation $\mathcal{T}_{\widetilde{\mathcal{S}}_{\text {reg }}}=\sigma\left(\mathcal{T}_{\left(K^{*} \backslash \mathrm{O}\right)}\right)$ of $\widetilde{\mathcal{S}}_{\text {reg. }}$. The triangulation $\mathcal{T}_{\widetilde{\mathcal{S}}_{\text {reg }}}$ has 4 vertices, corresponding to the $G$ orbits $\sigma\left(\rho\left(\mathcal{O}\left(D^{\prime}\right)\right)\right), \sigma\left(\rho\left(\mathcal{O}\left(\overline{D^{\prime}}\right)\right)\right), \sigma(\rho(\mathcal{O}(C)))$, and $\sigma\left(\rho\left(\mathcal{O}\left(D^{\prime} \& \overline{D^{\prime}}\right)\right)\right) ; 18$ open edges corresponding to $\sigma\left(\rho\left(R^{j}(O C)\right)\right), \sigma\left(\rho\left(R^{j}\left(O D^{\prime}\right)\right)\right)$, and $\sigma\left(\rho\left(R^{j}\left(C D^{\prime}\right)\right)\right)$ for $j=0,1, \ldots, 5$; and 12 open triangles $\sigma\left(\rho\left(R^{j}\left(\triangle O C D^{\prime}\right)\right)\right)$ and $\sigma\left(\rho\left(R^{j}\left(\triangle O C \overline{D^{\prime}}\right)\right)\right)$ for $j=0,1, \ldots, 5$. Thus, the Euler characteristic $\chi\left(\widetilde{\mathcal{S}}_{\text {reg }}\right)$ of $\widetilde{\mathcal{S}}_{\text {reg }}$ is $4-18+12=-2$. Since $\widetilde{\mathcal{S}}_{\text {reg }}$ is a 2-dimensional smooth real manifold, $\chi\left(\widetilde{\mathcal{S}}_{\text {reg }}\right)=2-2 g$, where $g$ is the genus of $\widetilde{\mathcal{S}}_{\text {reg. }}$. Hence $g=2$. So $\widetilde{\mathcal{S}}_{\text {reg }}$ is a smooth 2-sphere with 2 handles, less a finite number of points, which lies in a compact topological space $\widetilde{S}=\operatorname{cl}\left(K^{*}\right)^{\sim} / G$, that is its closure, see Figure 8. This completes the example.

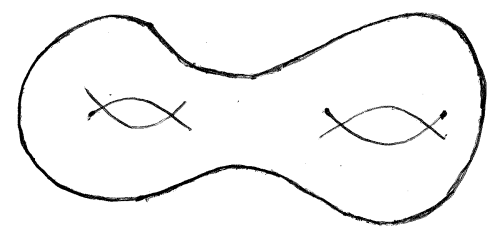

Figure 8. The G-orbit space $\widetilde{\mathcal{S}}_{\text {reg }}$ is 2-sphere with two handles.

Proof of Theorem 2. We now begin the construction of $\widetilde{\mathcal{S}}_{\text {reg }}$ by identifying equivalent edges of $\operatorname{cl}\left(K^{*}\right)$. For each $j=0,1, \infty$ let $\left[E, S_{m}^{(j)}(E)\right]$ be an unordered pair of equivalent closed edges of $\operatorname{cl}\left(K^{*}\right)$. We say that $x$ and $y$ in $\operatorname{cl}\left(K^{*}\right)$ are equivalent, $x \sim y$, if 1$) x$ and $y$ lie in $\partial \mathrm{cl}\left(K^{*}\right)$ with $x \in E$ and $y=S_{m}^{(j)}(x) \in S_{m}^{(0)}(E)$ for some $m \in\{0,1, \ldots, n-1\}$ and some $j=0,1, \infty$ or 2) $x$ and $y$ lie in int $\operatorname{cl}\left(K^{*}\right)$ and $x=y$. The relation $\sim$ is an equivalence relation on $\operatorname{cl}\left(K^{*}\right)$. Let $\operatorname{cl}\left(K^{*}\right)^{\sim}$ be the set of equivalence classes and let

$$
\rho: \operatorname{cl}\left(K^{*}\right) \rightarrow \operatorname{cl}\left(K^{*}\right)^{\sim}: p \mapsto[p]
$$

be the map which sends $p$ to the equivalence class $[p]$, that contains $p$. Compare this argument with that of Richens and Berry [2]. Give $\mathrm{cl}\left(K^{*}\right)$ the topology induced from $\mathbb{C}$ and put the quotient topology on $\mathrm{cl}\left(K^{*}\right)^{\sim}$.

Theorem 3. Let $K^{*}$ be $\mathrm{cl}\left(K^{*}\right)$ less its vertices. Then $\left(K^{*} \backslash \mathrm{O}\right)^{\sim}=\rho\left(K^{*} \backslash \mathrm{O}\right)$ is a smooth manifold. Furthermore, $\mathrm{cl}\left(K^{*}\right) \sim$ is a topological manifold.

Proof. To show that $\left(K^{*} \backslash \mathrm{O}\right) \sim$ is a smooth manifold, let $E_{+}$be an open edge of $K^{*}$. For $p_{+} \in E_{+}$let $D_{p_{+}}$be a disk in $\mathbb{C}$ with center at $p_{+}$, which does not contain a vertex of 
$\operatorname{cl}\left(K^{*}\right)$. Set $D_{p_{+}}^{+}=K^{*} \cap D_{p_{+}}$. For each $j=0,1, \infty$ let $E_{-}$be an open edge of $K^{*}$, which is equivalent to $E_{+}$via the reflection $S_{m}^{(j)}$, that is, $\left[\mathrm{cl}\left(E_{+}\right), \operatorname{cl}\left(E_{-}\right)=S_{m}^{(j)}\left(\operatorname{cl}\left(E_{+}\right)\right)\right] \in \mathcal{E}^{j}$ is an unordered pair of $S_{m}^{(j)}$ equivalent edges. Let $p_{-}=S_{m}^{(j)}\left(p_{+}\right)$and set $D_{p_{-}}^{-}=S_{m}^{(j)}\left(D_{p_{+}}^{+}\right)$. Then $V_{[p]}=\rho\left(D_{p_{+}}^{+} \cup D_{p_{-}}^{-}\right)$is an open neighborhood of $[p]=\left[p_{+}\right]=\left[p_{-}\right]$in $\left(K^{*} \backslash \mathrm{O}\right)^{\sim}$, which is a smooth 2-disk, since the identification mapping $\rho$ is the identity on int $K^{*}$. It follows that $\left(K^{*} \backslash \mathrm{O}\right)^{\sim}$ is a smooth 2-dimensional manifold without boundary.

We now handle the vertices of $\mathrm{cl}\left(K^{*}\right)$. Let $v_{+}$be a vertex of $\operatorname{cl}\left(K^{*}\right)$ and set $D_{v_{+}}=$ $\widetilde{D} \cap \operatorname{cl}\left(K^{*}\right)$, where $\widetilde{D}$ is a disk in $\mathbb{C}$ with center at the vertex $v_{+}=r_{0} \mathrm{e}^{i \pi \theta_{0}}$. The map

$$
W_{v_{+}}: D_{+} \subseteq \mathbb{C} \rightarrow D_{v_{+}} \subseteq \mathbb{C}: r \mathrm{e}^{i \pi \theta} \mapsto\left|r-r_{0}\right| \mathrm{e}^{i \pi s\left(\theta-\theta_{0}\right)}
$$

with $r \geq 0$ and $0 \leq \theta \leq 1$ is a homeomorphism, which sends the wedge with angle $\pi$ to the wedge with angle $\pi s$. The latter wedge is formed by the closed edges $E_{+}^{\prime}$ and $E_{+}$ of $\mathrm{cl}\left(K^{*}\right)$, which are adjacent at the vertex $v_{+}$such that $\mathrm{e}^{i \pi s} E_{+}^{\prime}=E_{+}$with the edge $E_{+}^{\prime}$ being swept out through int $\mathrm{cl}\left(K^{*}\right)$ during its rotation to the edge $E_{+}$. Because $\operatorname{cl}\left(K^{*}\right)$ is a rational regular stellated $n$-gon, the value of $s$ is a rational number for each vertex of $\mathrm{cl}\left(K^{*}\right)$. For each $j=0,1, \infty$ let $E_{-}=S_{m}^{(j)}\left(E_{+}\right)$be an edge of $\mathrm{cl}\left(K^{*}\right)$, which is equivalent to $E_{+}$and set $v_{-}=S_{m}^{(j)}\left(v_{+}\right)$. Then $v_{-}$is a vertex of $\mathrm{cl}\left(K^{*}\right)$, which is the center of the disk $D_{v_{-}}=S_{m}^{(j)}\left(D_{v_{+}}\right)$. Set $D_{-}=\bar{D}_{+}$. Then $D=D_{+} \cup D_{-}$is a disk in $\mathbb{C}$. The map $W: D \rightarrow$ $\rho\left(D_{v_{+}} \cup D_{v_{-}}\right)$, where $\left.W\right|_{D_{+}}=\rho \circ W_{v_{+}}$and $\left.W\right|_{D_{-}}=\rho \circ S_{m}^{(0)} \circ W_{v_{+}} \circ^{-}$, is a homeomorphism of $D$ into a neighborhood $\rho\left(D_{v_{+}} \cup D_{v_{-}}\right)$of $[v]=\left[v_{+}\right]=\left[v_{-}\right]$in $\mathrm{cl}\left(K^{*}\right)^{\sim}$. Consequently, the identification space $\mathrm{cl}\left(K^{*}\right) \sim$ is a topological manifold.

We now describe a triangulation of $K^{*} \backslash \mathrm{O}$. Let $T^{\prime}=T_{1, n_{1}, n-\left(1+n_{1}\right)}$ be the open rational triangle $\triangle O C D^{\prime}$ with vertex at the origin $O$, longest side $O C$ on the real axis, and interior angles $\frac{1}{n} \pi, \frac{n_{1}}{n} \pi$, and $\frac{n-1-n_{1}}{n} \pi$. Let $Q^{\prime}$ be the quadrilateral $T^{\prime} \cup \overline{T^{\prime}}$. Then $Q^{\prime}$ is a subset of the quadrilateral $Q=O D C \bar{D}$, see Figure 5. Moreover $K^{*}=\bigcup_{\ell=0}^{n-1} R^{\ell}\left(Q^{\prime}\right)$. The $2 n$ triangles $\operatorname{cl}\left(R^{j}\left(T^{\prime}\right)\right) \backslash\{O\}$ and $\operatorname{cl}\left(R^{k}\left(\overline{T^{\prime}}\right)\right) \backslash O$ with $k=0,1, \ldots, n-1$ form a triangulation $\mathcal{T}_{K^{*} \backslash \mathrm{O}}$ of $K^{*} \backslash \mathrm{O}$ with $2 n$ vertices $R^{k}(C)$ and $R^{k}\left(D^{\prime}\right)$ for $k=0,1, \ldots, n-1 ; 4 n$ open edges $R^{k}(O C), R^{k}\left(O D^{\prime}\right), R^{k}\left(C D^{\prime}\right)$, and $R^{k}\left(C \overline{D^{\prime}}\right)$ for $k=0,1, \ldots, n-1$; and $2 n$ open triangles $R^{k}\left(T^{\prime}\right), R^{k}\left(\overline{T^{\prime}}\right)$ with $k=0,1, \ldots, n-1$. The image of the triangulation $\mathcal{T}_{K^{*} \backslash \mathrm{O}}$ under the identification map $\rho$ (21) is a triangulation $\mathcal{T}_{\left(K^{*} \backslash \mathrm{O}\right)^{\sim}}$ of the identification space $\left(K^{*} \backslash \mathrm{O}\right)^{\sim}$.

The action of $G$ on $\mathrm{cl}\left(K^{*}\right)$ preserves the set of unordered pairs of $S_{m}^{(j)}$ equivalent edges of $\operatorname{cl}\left(K^{*}\right)$ for each $j=0,1, \infty$. Hence $G$ induces an action on $\mathrm{cl}\left(K^{*}\right)^{\sim}$, which is proper, since $G$ is finite. The $G$ action is free on $K^{*} \backslash O$ and thus on $\left(K^{*} \backslash O\right) \sim$ by Lemma A2. We have proved

Lemma 6. The G-orbit space $\widetilde{\mathcal{S}}=\mathrm{cl}\left(K^{*}\right)^{\sim} / G$ is a compact connected topological manifold with $\widetilde{\mathcal{S}}_{\text {reg }}=\left(K^{*} \backslash \mathrm{O}\right) \sim / \mathrm{G}$ being a smooth manifold. Let

$$
\sigma: \operatorname{cl}\left(K^{*}\right)^{\sim} \rightarrow \widetilde{\mathcal{S}}=\operatorname{cl}\left(K^{*}\right)^{\sim} / G: z \mapsto z G .
$$

Then $\sigma$ is the $G$ orbit map, which is surjective, continuous, and open. The restriction of $\sigma$ to $K^{*} \backslash \mathrm{O}$ has image $\widetilde{\mathcal{S}}_{\text {reg }}$ and is a smooth open mapping.

We now determine the topology of the orbit space $\widetilde{\mathcal{S}}_{\text {reg. }}$. For each $j=0,1, \infty$ and $\ell_{j}=0,1, \ldots, d_{j}-1$ let $A_{\ell_{j}}^{j}$ be an end point of a closed edge $E$ of $\mathrm{cl}\left(K^{*}\right)$, which lies on the unordered pair $\left[E, S_{\ell_{j}}^{(j)}(E)\right] \in \mathcal{E}^{j}$. Then $H^{j} \cdot A_{\ell_{j}}^{(j)}$ is an end point of the edge $H^{j} \cdot E$ of the unordered pair $H^{j} \cdot\left[E, S_{\ell_{j}}^{(j)}(E)\right]$ of $\mathcal{E}^{j}$. See Appendix A for the definition of the group $H_{j}$. The sets $\mathcal{O}\left(A_{\ell}^{(j)}\right)=\left\{H^{j} \cdot A_{\ell_{j}}^{(j)}\right\}$ with $\ell_{j}=0,1, \ldots, d_{j}-1$ are permuted by $G$. The action of $G$ on $K^{*} \backslash O$ preserves the set of open edges of the triangulation $\mathcal{T}_{K^{*} \backslash \mathrm{O}}$. There are 
3n-orbits: $R^{k}(O C) ; R^{k}\left(O \overline{D^{\prime}}\right)$, since $O D^{\prime}=R\left(O \overline{D^{\prime}}\right)$; and $R^{k}(C D)$, since $C \overline{D^{\prime}}=U(C D)$ for $k=0,1, \ldots, n-1$. So the image of the triangulation $\mathcal{T}_{K^{*} \backslash \mathrm{O}}$ under the continuous open map

$$
\mu=\left.\sigma \circ \pi\right|_{K^{*} \backslash \mathrm{O}}: K^{*} \backslash \mathrm{O} \rightarrow \widetilde{\mathcal{S}}_{\text {reg }}
$$

is a triangulation $\mathcal{T}_{\widetilde{\mathcal{S}}_{\text {reg }}}$ of the G-orbit space $\widetilde{\mathcal{S}}_{\text {reg }}$ with $d_{0}+d_{1}+d_{\infty}$ vertices $\mu\left(\mathcal{O}\left(A_{\ell_{j}}^{(j)}\right)\right)$, where $j=0,1, \infty$ and $\ell_{j}=0,1, \ldots, d_{j}-1$; $3 n$ open edges $\mu\left(R^{k}(O C)\right), \mu\left(R^{j}\left(O \overline{D^{\prime}}\right)\right)$, and $\mu\left(R^{k}(C D)\right)$ for $k=0,1, \ldots, n-1$; and $2 n$ open triangles $\mu\left(R^{k}\left(T^{\prime}\right)\right)$ and $\mu\left(R^{k}\left(\overline{T^{\prime}}\right)\right)$ for $k=0,1, \ldots n-1$. Thus, the Euler characteristic $\chi\left(\widetilde{\mathcal{S}}_{\text {reg }}\right)$ of $\widetilde{\mathcal{S}}_{\text {reg }}$ is $d_{0}+d_{1}+d_{\infty}-3 n+2 n=$ $d_{0}+d_{1}+d_{\infty}-n$. However, $\widetilde{\mathcal{S}}_{\text {reg }}$ is a smooth manifold. So $\chi\left(\widetilde{\mathcal{S}}_{\text {reg }}\right)=2-2 g$, where $g$ is the genus of $\widetilde{\mathcal{S}}_{\text {reg. }}$. Hence $g=\frac{1}{2}\left(n+2-\left(d_{0}+d_{1}+d_{\infty}\right)\right)$. Compare this argument with that of Weyl ([4], p. 174). This proves Theorem 2.

Since the quadrilateral $Q$ is a fundamental domain for the action of $G$ on $K^{*}$, the $G$ orbit map $\bar{\mu}=\sigma \circ \pi: K^{*} \subseteq \mathbb{C} \rightarrow \widetilde{\mathcal{S}}$ restricted to $Q$ is a bijective continuous open mapping. However, $\delta_{Q}: \mathcal{D} \subseteq \mathcal{S} \rightarrow Q \subseteq \mathbb{C}$ is a bijective continous open mapping of the fundamental domain $\mathcal{D}$ of the $\mathcal{G}$ action on $\mathcal{S}$. Consequently, the $\mathcal{G}$ orbit space is homeomorphic to the $G$ orbit space $\widetilde{\mathcal{S}}$. The mapping $\bar{\mu}$ is holomorphic except possibly at 0 and the vertices of $\mathrm{cl}\left(K^{*}\right)$. So the mapping $\bar{\mu} \circ \delta_{K^{*}}: \mathcal{S}_{\text {reg }} \rightarrow \widetilde{\mathcal{S}}_{\text {reg }}$ is a holomorphic diffeomorphism.

\section{An Affine Model of $\mathcal{S}_{\text {reg }}$}

We construct an affine model of the affine Riemann surface $\mathcal{S}_{\text {reg }}$ as follows. Return to the regular stellated $n$-gon $K^{*}=K_{n_{0} n_{1} n_{\infty}}^{*}$, which is formed from the quadrilateral $Q=$ $Q_{n_{0} n_{1} n_{\infty}}$ less its vertices. Repeatedly reflecting in the edges of $K^{*}$ and then in the edges of the resulting reflections of $K^{*}$ et cetera, we obtain a covering of $\mathbb{C} \backslash \mathbb{V}^{+}$by certain translations of $K^{*}$. Here $\mathbb{V}^{+}$is the union of the translates of the vertices of $\mathrm{cl}\left(K^{*}\right)$ and its center $O$. Let $\mathfrak{T}$ be the group generated by these translations. The semidirect product $\mathfrak{G}=G \ltimes \mathfrak{T}$ acts freely, properly and transitively on $\mathbb{C} \backslash \mathbb{V}^{+}$. It preserves equivalent edges of $\mathbb{C} \backslash \mathbb{V}^{+}$and it acts freely and properly on $\left(\mathbb{C} \backslash \mathbb{V}^{+}\right)^{\sim}$, the space formed by identifying equivalent edges in $\mathbb{C} \backslash \mathbb{V}^{+}$. The orbit space $\left(\mathbb{C} \backslash \mathbb{V}^{+}\right)^{\sim} / \mathfrak{G}$ is holomorphically diffeomorphic to $\widetilde{\mathcal{S}}_{\text {reg }}$ and is the desired affine model of $\mathcal{S}_{\text {reg. }}$. We now justify these assertions.

First we determine the group $\mathcal{T}$ of translations.

Lemma 7. Each of the $2 n$ sides of the regular stellated $n$-gon $K^{*}$ is perpendicular to exactly one of the directions

$$
\mathrm{e}^{\left[\frac{1}{2}-\frac{n_{1}}{n}+2 k \frac{1}{n}\right] \pi i} \text { or } \mathrm{e}^{\left[-\frac{1}{2}-\frac{1}{n}+\frac{n_{1}}{n}+(2 k+1) \frac{1}{n}\right] \pi i},
$$

for $k=0,1, \ldots, n-1$.

Proof. From Figure 9 we have $\angle D^{\prime} C O=\frac{n_{1}}{n} \pi$. So $\angle C O H=\frac{1}{2} \pi-\frac{n_{1}}{n} \pi$. Hence the line $\ell_{0}$, containing the edge $C D^{\prime}$ of $K^{*}$, is perpendicular to the direction $\mathrm{e}^{\left[\frac{1}{2}-\frac{n_{1}}{n}\right] \pi}$. Since $\triangle C O \overline{D^{\prime}}$ is the reflection of $\triangle C O D^{\prime}$ in the line segment $O C$, the line $\ell_{1}$, containing the edge $C \overline{D^{\prime}}$ of $K^{*}$, is perpendicular to the direction $\mathrm{e}^{\left[-\frac{1}{2}+\frac{n_{1}}{n}\right] \pi}$. Because the regular stellated $n$-gon $K^{*}$ is formed by repeatedly rotating the quadrilateral $Q^{\prime}=O D^{\prime} C \overline{D^{\prime}}$ through an angle $\frac{2 \pi}{n}$, we find that Equation (23) holds. 


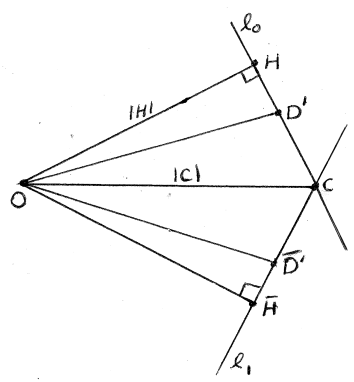

Figure 9. The regular stellated $n$-gon $K^{*}$ two of whose sides are $C D^{\prime}$ and $C \overline{D^{\prime}}$.

Since $\angle C O H=\frac{1}{2} \pi-\frac{n_{1}}{n} \pi$, it follows that $|H|=|C| \sin \pi \frac{n_{1}}{n}$ is the distance from the center $O$ of $K^{*}$ to the line $\ell_{0}$ containing the side $C D^{\prime}$, or to the line $\ell_{1}$ containing the side $C \overline{D^{\prime}}$. So $u_{0}=\left(|C| \sin \pi \frac{n_{1}}{n}\right) \mathrm{e}^{\left[\frac{1}{2}-\frac{n_{1}}{n}\right] \pi i}$ is the closest point $H$ on $\ell_{0}$ to $O$ and $u_{1}=$ $\left(|C| \sin \pi \frac{n_{1}}{n}\right) \mathrm{e}^{\left[-\frac{1}{2}+\frac{n_{1}}{n}\right] \pi i}$ is the closest point $\bar{H}$ on $\ell_{1}$ to $O$. Since the regular stellated $n$-gon $K^{*}$ is formed by repeatedly rotating the quadrilateral $Q^{\prime}=O D^{\prime} C \overline{D^{\prime}}$ through an angle $\frac{2 \pi}{n}$, the point

$$
u_{2 k}=R^{k} u_{0}=\left(|C| \sin \pi \frac{n_{1}}{n}\right) \mathrm{e}^{\left[\frac{1}{2}-\frac{n_{1}}{n}+2 k \frac{1}{n}\right] \pi i}
$$

lies on the line $\ell_{2 k}=R^{k} \ell_{0}$, which contains the edge $R^{k}\left(C D^{\prime}\right)$ of $K^{*}$; while

$$
u_{2 k+1}=R^{k} u_{1}=\left(|C| \sin \pi \frac{n_{1}}{n}\right) \mathrm{e}^{\left[-\frac{1}{2}+\frac{n_{1}}{n}-\frac{1}{n}+(2 k+1) \frac{1}{n}\right] \pi i}
$$

lies on the line $\ell_{2 k+1}=R^{k} \ell_{1}$, which contains the edge $R^{k}\left(C \overline{D^{\prime}}\right)$ of $K^{*}$ for every $k \in$ $\{0,1, \ldots, n-1\}$. Furthermore, the line segments $O u_{2 k}$ and $O u_{2 k+1}$ are perpendicular to the line $\ell_{2 k}$ and $\ell_{2 k+1}$, respectively, for $k \in\{0,1, \ldots, n-1\}$.

Corollary 8. For $k=0,1, \ldots, n-1$ we have

$$
\overline{u_{2 k}}=u_{2(n-k)+1} \text { and } \overline{u_{2 k+1}}=u_{2(n-k)} .
$$

Proof. We compute. From (24) it follows that

$$
\begin{aligned}
\overline{u_{2 k}} & =U\left(u_{2 k}\right)=U R^{k}\left(u_{0}\right)=R^{-k}\left(U\left(u_{0}\right)\right) \\
& =R^{-k}\left(u_{1}\right)=R^{n-k}\left(u_{1}\right)=u_{2(n-k)+1}, \quad \text { using (25); }
\end{aligned}
$$

while from (25) we get

$$
\overline{u_{2 k+1}}=U\left(u_{2 k+1}\right)=U R^{k}\left(u_{1}\right)=R^{-k}\left(U\left(u_{1}\right)\right)=R^{n-k}\left(u_{0}\right)=u_{2(n-k)} .
$$

Corollary 9. For $k, \ell \in\{0,1, \ldots, 2 n-1\}$ we have

$$
u_{(k+2 \ell) \bmod 2 n}=R^{\ell} u_{k} .
$$

Proof. If $k=2 i$, then $u_{k}=R^{i} u_{0}$, by definition. So

$$
R^{\ell} u_{k}=R^{\ell+i} u_{0}=u_{(2 i+2 \ell) \bmod 2 n}=u_{(k+2 \ell) \bmod 2 n} .
$$

If $k=2 i+1$, then $u_{\ell}=R^{i} u_{1}$, by definition. So

$$
R^{\ell} u_{k}=R^{\ell+i} u_{1}=u_{(2(i+\ell)+1) \bmod 2 n}=u_{(k+2 \ell) \bmod 2 n} .
$$


For $k=0,1, \ldots, 2 n-1$ let $\tau_{k}$ be the translation

$$
\tau_{k}: \mathbb{C} \rightarrow \mathbb{C}: z \mapsto z+2 u_{k}
$$

Corollary 10. For $k, \ell \in\{0,1, \ldots, 2 n-1\}$ we have

$$
\tau_{(k+2 \ell) \bmod 2 n^{\circ}} R^{\ell}=R^{\ell} \circ \tau_{k} .
$$

Proof. For every $z \in \mathbb{C}$, we have

$$
\begin{aligned}
\tau_{(k+2 \ell) \bmod 2 n}(z) & =z+2 u_{(k+2 \ell) \bmod 2 n,} \quad \text { using (28) } \\
& =z+2 R^{\ell} u_{k} \text { by (27) } \\
& =R^{\ell}\left(R^{-\ell} z+2 u_{k}\right)=R^{\ell} \circ \tau_{k}\left(R^{-\ell} z\right) .
\end{aligned}
$$

Reflecting the regular stellated $n$-gon $K^{*}$ in its edge $C D^{\prime}$ contained in $\ell_{0}$ gives a congruent regular stellated $n$-gon $K_{0}^{*}$ with the center $O$ of $K^{*}$ becoming the center $2 u_{0}$ of $K_{0}^{*}$.

Lemma 8. The collection of all the centers of the regular stellated $n$-gons, formed by reflecting $K^{*}$ in its edges and then reflecting in the edges of the reflected regular stellated n-gons et cetera, is

$$
\begin{aligned}
\left\{\tau_{0}^{\ell_{0}} \circ \cdots \circ \tau_{2 n-1}^{\ell_{2 n-1}}(0)\right. & \left.\in \mathbb{C} \mid\left(\ell_{0}, \ldots, \ell_{2 n-1}\right) \in\left(\mathbb{Z}_{\geq 0}\right)^{2 n}\right\}= \\
= & \left\{2 \sum_{\ell_{0}, \ldots, \ell_{2 n-1}=0}^{\infty}\left(\ell_{0} u_{0}+\cdots \ell_{2 n-1} u_{2 n-1}\right)\right\},
\end{aligned}
$$

where for $k=0,1, \ldots, 2 n-1$ we have

$$
\tau_{k}^{\ell_{k}}=\overbrace{\tau_{k^{\circ}} \cdots \circ \tau_{k}}^{\ell_{k}}: \mathbb{C} \rightarrow \mathbb{C}: z \mapsto z+2 \ell_{j} u_{k} .
$$

Proof. For each $k_{0}=0,1, \ldots, 2 n-1$ the center of the $2 n$ regular stellated congruent $n$ gon $K_{k_{0}}^{*}$ formed by reflecting in an edge of $K^{*}$ contained in the line $\ell_{k_{0}}$ is $\tau_{k_{0}}(0)=2 u_{k_{0}}$. Repeating the reflecting process in each edge of $K_{k_{0}}^{*}$ gives $2 n$ congruent regular stellated $n$ gons $K_{k_{0} k_{1}}^{*}$ with center at $\tau_{k_{1}}\left(\tau_{k_{0}}(0)\right)=2\left(u_{k_{1}}+u_{k_{0}}\right)$, where $k_{1}=0,1, \ldots 2 n-1$. Repeating this construction proves the lemma.

The set $\mathbb{V}$ of vertices of the regular stellated $n$-gon $K^{*}$ is

$$
\left\{V_{2 k}=C \mathrm{e}^{2 k\left(\frac{1}{n} \pi i\right)}, V_{2 k+1}=D^{\prime} \mathrm{e}^{(2 k+1)\left(\frac{1}{n} \pi i\right)} \text { for } 0 \leq k \leq n-1\right\},
$$

see Figure 5. Clearly the set $\mathbb{V}$ is $G$ invariant.

Corollary 11. The set

$$
\begin{aligned}
\mathbb{V}^{+}=\left\{v_{\ell_{0} \cdots \ell_{2 n-1}}=\tau_{0}^{\ell_{0}} \circ \cdots \circ \tau_{2 n-1}^{\ell_{2 n-1}}(V) \mid\right. \\
\left.V \in \mathbb{V} \cup\{\mathrm{O}\} \&\left(\ell_{0}, \ldots, \ell_{2 n-1}\right) \in\left(\mathbb{Z}_{\geq 0}\right)^{2 n}\right\}
\end{aligned}
$$

is the collection of vertices and centers of the congruent regular stellated $n$-gons $K^{*}, K_{k_{1}}^{*}, K_{k_{0} k_{1}}^{*}, \ldots$

Proof. This follows immediately from Lemma 8. 
Corollary 12. The union of $K^{*}, K_{k_{0}}^{*}, K_{k_{0} k_{1}}^{*}, \ldots K_{k_{0} k_{1} \cdots k_{\ell}}^{*} \ldots$, where $\ell \geq 0,0 \leq j \leq \ell$, and $0 \leq k_{j} \leq 2 n-1$, covers $\mathbb{C} \backslash \mathbb{V}^{+}$, that is,

$$
K^{*} \cup \bigcup_{\ell \geq 0} \bigcup_{0 \leq j \leq \ell} \bigcup_{0 \leq k_{j} \leq 2 n-1} K_{k_{0} k_{1} \cdots k_{\ell}}^{*}=\mathbb{C} \backslash \mathbb{V}^{+} .
$$

Proof. This follows immediately from $K_{k_{0} k_{1} \cdots k_{\ell}}^{*}=\tau_{k_{\ell}} \circ \cdots \circ \tau_{k_{0}}\left(K^{*}\right)$.

Let $\mathcal{T}$ be the abelian subgroup of the 2-dimensional Euclidean group $\mathrm{E}(2)$ generated by the translations $\tau_{k}$ (28) for $k=0,1, \ldots 2 n-1$. It follows from Corollary 12 that the regular stellated $n$-gon $K^{*}$ with its vertices and center removed is the fundamental domain for the action of the abelian group $\mathcal{T}$ on $\mathbb{C} \backslash \mathbb{V}^{+}$. The group $\mathcal{T}$ is isomorphic to the abelian subgroup $\mathfrak{T}$ of $(\mathbb{C},+)$ generated by $\left\{2 u_{k}\right\}_{k=0}^{2 n-1}$.

Next we define the group $\mathfrak{G}$ and show that it acts freely, properly, and transitively on $\mathbb{C} \backslash \mathbb{V}^{+}$. Consider the group $\mathfrak{G}=G \ltimes \mathfrak{T} \subseteq G \times \mathfrak{T}$, which is the semidirect product of the dihedral group $G$, generated by the rotation $R$ through $2 \pi / n$ and the reflection $U$ subject to the relations $R^{n}=e=U^{2}$ and $R U=U R^{-1}$, and the abelian group $\mathfrak{T}$. An element $\left(R^{j} U^{\ell}, 2 u_{k}\right)$ of $\mathfrak{G}$ is the affine linear map

$$
\left(R^{j} U^{\ell}, 2 u_{k}\right): \mathbb{C} \rightarrow \mathbb{C}: z \mapsto R^{j} U^{\ell} z+2 u_{k} .
$$

Multiplication in $\mathfrak{G}$ is defined by

$$
\left(R^{j} U^{\ell}, 2 u_{k}\right) \cdot\left(R^{j^{\prime}} U^{\ell^{\prime}}, 2 u_{k^{\prime}}\right)=\left(R^{j+j^{\prime}} U^{\ell+\ell^{\prime}},\left(R^{j} U^{\ell}\right)\left(2 u_{k^{\prime}}\right)+2 u_{k}\right),
$$

which is the composition of the affine linear map $\left(R^{j^{\prime}} U^{\ell^{\prime}}, 2 u_{k^{\prime}}\right)$ followed by $\left(R^{j} U^{\ell}, 2 u_{k}\right)$. The mappings $G \rightarrow \mathfrak{G}: R^{j} \mapsto\left(R^{j} U^{\ell}, 0\right)$ and $\mathfrak{T} \rightarrow \mathfrak{G}: 2 u_{k} \mapsto\left(e, 2 u_{k}\right)$ are injective, which allows us to identify the groups $G$ and $\mathfrak{T}$ with their image in $\mathfrak{G}$. Using (31) we may write an element $\left(R^{j} U^{\ell}, 2 u_{k}\right)$ of $\mathfrak{G}$ as $\left(e, 2 u_{k}\right) \cdot\left(R^{j} U^{\ell}, 0\right)$. So

$$
\left(e, 2 u_{(j+2 k) \bmod 2 n}\right) \cdot\left(R^{k} U^{\ell}, 0\right)=\left(R^{k} U^{\ell}, 2 u_{(j+2 k) \bmod 2 n}\right),
$$

For every $z \in \mathbb{C}$ we have

$$
R^{k} U^{\ell} z+2 u_{(j+2 k) \bmod 2 n}=R^{k} U^{\ell} z+R^{k} U^{\ell}\left(2 u_{j}\right), \quad \text { using (27), }
$$

that is,

$$
\left(R^{k} U^{\ell}, 2 u_{(j+2 k) \bmod 2 n}\right)=\left(R^{k} U^{\ell}, R^{k} U^{\ell}\left(2 u_{j}\right)\right)=\left(R^{k} U^{\ell}, 0\right) \cdot\left(e, 2 u_{j}\right) .
$$

Hence

$$
\left(e, 2 u_{(j+2 k) \bmod 2 n}\right) \cdot\left(R^{k} U^{\ell}, 0\right)=\left(R^{k} U^{\ell}, 0\right) \cdot\left(e, 2 u_{j}\right),
$$

which is just Equation (29). The group $\mathfrak{G}$ acts on $\mathbb{C}$ as $E(2)$ does, namely, by affine linear orthogonal mappings. Denote this action by

$$
\psi: \mathfrak{G} \times \mathbb{C} \rightarrow \mathbb{C}:((g, \tau), z) \mapsto \tau(g(z)) .
$$

Lemma 9. The set $\mathbb{V}^{+}(30)$ is invariant under the $\mathfrak{G}$ action.

Proof. Let $v \in \mathbb{V}^{+}$. Then for some $\left(\ell_{0}^{\prime}, \ldots, \ell_{2 n-1}^{\prime}\right) \in \mathbb{Z}_{\geq 0}^{2 n}$ and some $w \in \mathbb{V} \cup\{\mathrm{O}\}$

$$
v=\tau_{0}^{\ell_{0}^{\prime}} \cdots \circ \tau_{2 n-1}^{\ell_{2 n-1}^{\prime}}(w)=\psi_{\left(e, 2 u^{\prime}\right)}(w),
$$

where $u^{\prime}=\sum_{k=0}^{2 n-1} \ell_{k}^{\prime} u_{k}$. For $\left(R^{j} U^{\ell}, 2 u\right) \in \mathfrak{G}$ with $j=0,1, \ldots, n-1$ and $\ell=0,1$ we have

$$
\psi_{\left(R^{j} U^{\ell}, 2 u\right)^{v}} v=\psi_{\left(R^{j} U^{\ell}, 2 u\right)^{\circ}} \psi_{\left(e, 2 u^{\prime}\right)}(w)=\psi_{\left(R^{j} U^{\ell}, 2 u\right) \cdot\left(e, 2 u^{\prime}\right)}(w)
$$




$$
\begin{aligned}
& =\psi_{\left(R^{j} U^{\ell}, R^{j} U^{\ell}\left(2 u^{\prime}\right)+2 u\right)}(w)=\psi_{\left(e, 2\left(R^{j} U^{\ell} u^{\prime}+u\right)\right) \cdot\left(R^{j} U^{\ell}, 0\right)}(w) \\
& =\psi_{\left(e, 2\left(R^{j} U^{\ell} u^{\prime}+u\right)\right)}\left(\psi_{\left(R^{j} U^{\ell}, 0\right)}(w)\right)=\psi_{\left(e, 2\left(R^{j} U^{\ell} u^{\prime}+u\right)\right)}\left(w^{\prime}\right),
\end{aligned}
$$

where $w^{\prime}=\psi_{\left(R^{j} U^{\ell}, 0\right)}(w)=R^{j} U^{\ell}(w) \in \mathbb{V} \cup\{\mathrm{O}\}$. If $\ell=0$, then

$$
R^{j} u^{\prime}=R^{j}\left(\sum_{k=0}^{2 n-1} \ell_{k}^{\prime} u_{k}\right)=\sum_{k=0}^{2 n-1} \ell_{k}^{\prime} R^{j}\left(u_{k}\right)=\sum_{k=0}^{2 n-1} \ell_{k}^{\prime} u_{(k+2 j) \bmod 2 n} ;
$$

while if $\ell=1$, then

$$
R^{j} U\left(u^{\prime}\right)=\sum_{k=0}^{2 n-1} \ell_{k}^{\prime} R^{j}\left(U\left(u_{k}\right)\right)=\sum_{k=0}^{2 n-1} \ell_{k}^{\prime} R^{j}\left(u_{k^{\prime}(k)}\right)=\sum_{k=0}^{2 n-1} \ell_{k}^{\prime} u_{\left(k^{\prime}(k)+2 j\right) \bmod 2 n} .
$$

Here $k^{\prime}(k)=\left\{\begin{array}{l}2 n-k+1, \text { if } k \text { is even } \\ 2 n-k-1 \text { if } k \text { is odd, }\end{array}\right.$ see Corollary 8. So $\left(e, 2\left(R^{j} U^{\ell} u^{\prime}+u\right)\right) \in \mathfrak{T}$, which implies $\psi_{\left(e, 2\left(R i U^{\ell} u^{\prime}+u\right)\right)}\left(w^{\prime}\right) \in \mathbb{V}^{+}$, as desired.

Lemma 10. The action of $\mathfrak{G}$ on $\mathbb{C} \backslash \mathbb{V}^{+}$is free.

Proof. Suppose that for some $v \in \mathbb{C} \backslash \mathbb{V}^{+}$and some $\left(R^{j} U^{\ell}, 2 u\right) \in \mathfrak{G}$ we have $v=$ $\psi_{\left(R^{j} U^{\ell}, 2 u\right)}(v)$. Then $v$ lies in some $K_{k_{0} k_{1} \cdots k_{\ell}}^{*}$. So for some $v^{\prime} \in K^{*}$ we have

$$
v=\tau_{0}^{\ell_{0}^{\prime}} \cdots \tau_{2 n-1}^{\ell_{2 n-1}^{\prime}}\left(v^{\prime}\right)=\psi_{\left(e, 2 u^{\prime}\right)}\left(v^{\prime}\right),
$$

where $u^{\prime}=\sum_{j=0}^{2 n-1} \ell_{j}^{\prime} u_{j}$ for some $\left(\ell_{0}^{\prime}, \ldots, \ell_{2 n-1}^{\prime}\right) \in\left(\mathbb{Z}_{\geq 0}\right)^{2 n}$. Thus,

$$
\psi_{\left(e, 2 u^{\prime}\right)}\left(v^{\prime}\right)=\psi_{\left(R^{j} U^{\ell}, 2 u\right) \cdot\left(e, 2 u^{\prime}\right)}\left(v^{\prime}\right)=\psi_{\left(R^{j} U^{\ell}, 2 R^{j} U^{\ell} u^{\prime}+2 u\right)}\left(v^{\prime}\right) .
$$

This implies $R^{j} U^{\ell}=e$, that is, $j=\ell=0$. So $2 u^{\prime}=2 R^{j} U^{\ell} u^{\prime}+2 u=2 u^{\prime}+2 u$, that is, $u=0$. Hence $\left(R^{j} U^{\ell}, u\right)=(e, 0)$, which is the identity element of $\mathfrak{G}$.

Lemma 11. The action of $\mathcal{T}$ (and hence $\mathfrak{G}$ ) on $\mathbb{C} \backslash \mathbb{V}^{+}$is transitive.

Proof. Let $K_{k_{0} \cdots k_{\ell}}^{*}$ and $K_{k_{0}^{\prime} \cdots k_{\ell^{\prime}}^{\prime}}^{*}$ lie in

$$
\mathbb{C} \backslash \mathbb{V}^{+}=K^{*} \cup \bigcup_{\ell \geq 0} \bigcup_{0 \leq j \leq \ell} \bigcup_{0 \leq k_{j} \leq 2 n-1} K_{k_{0} k_{1} \cdots k_{\ell}}^{*} .
$$

Since $K_{k_{0} \cdots k_{\ell}}^{*}=\tau_{k_{\ell}} \circ \cdots \circ \tau_{k_{0}}\left(K^{*}\right)$ and $K_{k_{0}^{\prime} \cdots k_{\ell^{\prime}}^{\prime}}^{*}=\tau_{k_{\ell^{\prime}}} \cdots \circ \tau_{k_{0}^{\prime}}\left(K^{*}\right)$, it follows that $\left(\tau_{k_{\ell^{\prime}}^{\prime}} \circ \cdots \circ \tau_{k_{0}^{\prime}}\right) \circ\left(\tau_{k_{\ell}} \circ \cdots \circ \tau_{k_{0}}\right)^{-1}\left(K_{k_{0} \cdots k_{\ell}}^{*}\right)=K_{k_{0}^{\prime} \cdots k_{\ell^{\prime}}^{\prime}}^{*} \cdot$

The action of $\mathfrak{G}$ on $\mathbb{C} \backslash \mathbb{V}^{+}$is proper because $\mathfrak{G}$ is a discrete subgroup of $\mathrm{E}(2)$ with no accumulation points.

We now define an edge of $\mathbb{C} \backslash \mathbb{V}^{+}$and what it means for an unordered pair of edges to be equivalent. We show that the group $\mathfrak{G}$ acts freely and properly on the identification space of equivalent edges.

Let $E$ be an open edge of $K^{*}$. Since $E_{k_{0} \cdots k_{\ell}}=\tau_{k_{0}} \cdots \tau_{k_{\ell}}(E) \in K_{k_{0} \cdots k_{\ell}}^{*}$ it follows that $E_{k_{0} \cdots k_{\ell}}$ is an open edge of $K_{k_{0} \cdots k_{\ell}}^{*}$. Let

$$
\mathfrak{E}=\left\{E_{k_{0} \cdots k_{\ell}} \mid \ell \geq 0,0 \leq j \leq \ell \& 0 \leq k_{j} \leq 2 n-1\right\} .
$$

Then $\mathfrak{E}$ is the set of open edges of $\mathbb{C} \backslash \mathbb{V}^{+}$by 12 . Since $\tau_{k_{\ell}} \circ \cdots \circ \tau_{k_{0}}(0)$ is the center of $K_{k_{0} \cdots k_{\ell^{\prime}}}^{*}$ the element $\left(e, \tau_{k_{\ell}} \circ \cdots \circ \tau_{k_{0}}\right) \cdot\left(g,\left(\tau_{k_{\ell}} \circ \cdots \circ \tau_{k_{0}}\right)^{-1}\right)$ of $\mathfrak{G}$ is a rotation-reflection of 
$K_{k_{0} \cdots k_{\ell}}^{*}$, which sends an edge of $K_{k_{0} \cdots k_{\ell}}^{*}$ to another edge of $g * K_{k_{0} \cdots k_{\ell}}^{*}$. Thus, $\mathfrak{G}$ sends $\mathfrak{E}$ into itself. For $j=0,1, \infty$ let $\mathfrak{E}_{k_{0} \cdots k_{\ell}}^{j}$ be the set of unordered pairs $\left[E_{k_{0} \cdots k_{\ell}}, E_{k_{0} \cdots k_{\ell}}^{\prime}\right]$ of equivalent open edges of $K_{k_{0} \cdots k_{\ell}}^{*}$, that is, $E_{k_{0} \cdots k_{\ell}} \cap E_{k_{0} \cdots k_{\ell}}^{\prime}=\varnothing$, so the open edges $E_{k_{0} \cdots k_{\ell}}=\tau_{k_{0}} \cdots \tau_{k_{\ell}}(E)$ and $E_{k_{0} \cdots k_{\ell}}^{\prime}=\tau_{k_{0}} \cdots \tau_{k_{\ell}}\left(E^{\prime}\right)$ of $\mathrm{cl}\left(K_{k_{0} \cdots k_{\ell}}^{*}\right)$ are not adjacent, which implies that the open edges $E$ and $E^{\prime}$ of $K^{*}$ are not adjacent, and for some generator $S_{m}^{(j)}$ of the group $G^{j}$ of reflections with $j=0,1, \infty$ we have

$$
E_{k_{0} \cdots k_{\ell}}^{\prime}=\left(\tau_{k_{0}} \circ \cdots \circ \tau_{k_{0}}\right)\left(S_{m}^{(j)}\left(\left(\tau_{k_{\ell}} \circ \cdots \circ \tau_{k_{0}}\right)^{-1}\left(E_{k_{0} \cdots k_{\ell}}\right)\right)\right) .
$$

Let $\mathfrak{E}^{j}=\cup_{\ell \geq 0} \cup_{0 \leq j \leq \ell} \cup_{0 \leq k_{j} \leq 2 n-1} \mathfrak{E}_{k_{0} \cdots k_{\ell}}^{j}$. So $\cup_{j=0,1, \infty} \mathfrak{E}^{j}$ is the set of unordered pairs of equivalent edges of $\mathbb{C} \backslash \mathbb{V}^{+}$. Define an action $*$ of $\mathfrak{G}$ on $\bigcup_{j=0,1, \infty} \mathcal{E}^{j}$ by

$$
\begin{aligned}
(g, \tau) *\left[E_{k_{0} \cdots k_{\ell}} E_{k_{0} \cdots k_{\ell}}^{\prime}\right] & =\left(\left[\left(\tau^{\prime} \circ \tau\right)\left(g\left(\tau^{\prime}\right)^{-1}\left(E_{k_{0} \cdots k_{\ell}}\right)\right),\left(\tau^{\prime} \circ \tau\right)\left(g\left(\left(\tau^{\prime}\right)^{-1}\left(E_{k_{0} \cdots k_{\ell}}^{\prime}\right)\right)\right]\right)\right. \\
& =\left[(g, \tau) * E_{k_{0} \cdots k_{\ell}}(g, \tau) * E_{k_{0} \cdots k_{\ell}}^{\prime}\right],
\end{aligned}
$$

where $\tau^{\prime}=\tau_{k_{\ell}} \circ \cdots \tau_{k_{0}}$.

Define a relation $\sim$ on $\mathbb{C} \backslash \mathbb{V}^{+}$as follows. We say that $x$ and $y \in \mathbb{C} \backslash \mathbb{V}^{+}$are related, $x \sim y$, if 1) $x \in F=\tau(E) \in \mathfrak{E}^{j}$ and $y \in F^{\prime}=\tau\left(E^{\prime}\right) \in \mathfrak{E}^{j}$ such that $\left[F, F^{\prime}\right]=\left[\tau(E), \tau\left(E^{\prime}\right)\right] \in$ $\mathfrak{E}^{0}$, where $\left[E, E^{\prime}\right] \in \mathcal{E}^{j}$ with $E^{\prime}=S_{m}^{(j)}(E)$ for some $S_{m}^{(j)} \in G^{j}$ and $y=\tau\left(S_{m}^{(j)}\left(\tau^{-1}(x)\right)\right)$ for some $j=0,1, \infty$, or 2) $x, y \in\left(\mathbb{C} \backslash \mathbb{V}^{+}\right) \backslash \mathfrak{E}$ and $x=y$. Then $\sim$ is an equivalence relation on $\mathbb{C} \backslash \mathbb{V}^{+}$. Let $\left(\mathbb{C} \backslash \mathbb{V}^{+}\right)^{\sim}$ be the set of equivalence classes and let $\Pi$ be the map

$$
\Pi: \mathbb{C} \backslash \mathbb{V}^{+} \rightarrow\left(\mathbb{C} \backslash \mathbb{V}^{+}\right)^{\sim}: p \mapsto[p],
$$

which assigns to every $p \in \mathbb{C} \backslash \mathbb{V}^{+}$the equivalence class $[p]$ containing $p$.

Lemma 12. $\left.\Pi\right|_{K^{*}}$ is the map $\rho(20)$.

Proof. This follows immediately from the definition of the maps $\Pi$ and $\rho$.

Lemma 13. The usual action of $\mathfrak{G}$ on $\mathbb{C}$, restricted to $\mathbb{C} \backslash \mathbb{V}^{+}$, is compatible with the equivalence relation $\sim$, that is, if $x, y \in \mathbb{C} \backslash \mathbb{V}$ and $x \sim y$, then $(g, \tau)(x) \sim(g, \tau)(y)$ for every $(g, \tau) \in \mathfrak{G}$.

Proof. Suppose that $x \in F=\tau^{\prime}(E)$, where $\tau^{\prime} \in \mathcal{T}$. Then $y \in F^{\prime}=\tau^{\prime}\left(E^{\prime}\right)$, since $x \sim y$. So for some $S_{m}^{(j)} \in G^{j}$ with $j=0,1, \infty$, we have $\left(\tau^{\prime}\right)^{-1}(y)=S_{m}^{(j)}\left(\tau^{-1}(x)\right)$. Let $(g, \tau) \in \mathfrak{G}$. Then

$$
(g, \tau)\left(\left(\tau^{\prime}\right)^{-1}(y)\right)=g\left(\left(\tau^{\prime}\right)^{-1}(y)\right)+u_{\tau}=g\left(S_{m}^{(j)}\left(\tau^{-1}(x)\right)\right)+u_{\tau} .
$$

So $(g, \tau)(y) \in(g, \tau) * F^{\prime}$. However, $(g, \tau)(x) \in(g, \tau) * F$ and $\left[(g, \tau) * F,(g, \tau) * F^{\prime}\right]=$ $(g, \tau) *\left[F, F^{\prime}\right]$. Hence $(g, \tau)(x) \sim(g, \tau)(y)$. $\left.\mathbb{V}^{+}\right)^{\sim}$.

Because of Lemma 13, the usual $\mathfrak{G}$-action on $\mathbb{C} \backslash \mathbb{V}^{+}$induces an action of $\mathfrak{G}$ on $(\mathbb{C} \backslash$

Lemma 14. The action of $\mathfrak{G}$ on $\left(\mathbb{C} \backslash \mathbb{V}^{+}\right)^{\sim}$ is free and proper.

Proof. The following argument shows that it is free. Using Lemma A2 we see that an element of $\mathfrak{G}$, which lies in the isotropy group $\mathfrak{G}_{\left[F, F^{\prime}\right]}$ for $\left[F, F^{\prime}\right] \in \mathfrak{E}^{0}$, interchanges the edge $F$ with the equivalent edge $F^{\prime}$ and thus fixes the equivalence class $[p]$ for every $p \in F$. Hence the $\mathfrak{G}$ action on $\left(\mathbb{C} \backslash \mathbb{V}^{+}\right)^{\sim}$ is free. It is proper because $\mathfrak{G}$ is a discrete subgroup of the Euclidean group E(2) with no accumulation points.

Theorem 4. The $\mathfrak{G}$-orbit space $\left(\mathbb{C} \backslash \mathbb{V}^{+}\right)^{\sim} / \mathfrak{G}$ is holomorphically diffeomorphic to the G-orbit space $\left(K^{*} \backslash \mathrm{O}\right)^{\sim} / G=\widetilde{\mathcal{S}}_{\text {reg }}$. 
Proof. The claim follows because the fundamental domain of the $\mathfrak{G}$-action on $\mathbb{C} \backslash \mathbb{V}^{+}$ is $K^{*} \backslash \mathrm{O}$ is the fundamental domain of the $G$-action on $K^{*} \backslash O$. Thus, $\Pi\left(\mathbb{C} \backslash \mathbb{V}^{+}\right)$is a fundamental domain of the $\mathfrak{G}$-action on $\left(\mathbb{C} \backslash \mathbb{V}^{+}\right)^{\sim}$, which is equal to $\rho\left(K^{*} \backslash \mathrm{O}\right)=\left(K^{*} \backslash \mathrm{O}\right)^{\sim}$ by Lemma 12. Hence the $\mathfrak{G}$-orbit space $\left(\mathbb{C} \backslash \mathbb{V}^{+}\right)^{\sim} / \mathfrak{G}$ is equal to the $G$-orbit space $\widetilde{\mathcal{S}}_{\text {reg. }}$. So the identity map from $\Pi\left(\mathbb{C} \backslash \mathbb{V}^{+}\right)$to $\left(K^{*} \backslash \mathrm{O}\right)^{\sim}$ induces a holomorphic diffeomorphism of orbit spaces.

Because the group $\mathfrak{G}$ is a discrete subgroup of the 2-dimensional Euclidean group $\mathrm{E}(2)$, the Riemann surface $\left(\mathbb{C} \backslash \mathbb{V}^{+}\right) \sim / \mathfrak{G}$ is an affine model of the affine Riemann surface $\mathcal{S}_{\text {reg. }}$.

\section{The Developing Map and Geodesics}

In this section, we show that the mapping

$$
\left.\delta: \mathcal{D} \subseteq \mathcal{S}_{\text {reg }} \rightarrow Q \subseteq \mathbb{C}:(\xi, \eta) \rightarrow\left(F_{Q} \circ \widehat{\pi}\right)(\xi, \eta)\right)
$$

straightens the holomorphic vector field $X$ (12) on the fundamental domain $\mathcal{D} \subseteq \mathcal{S}_{\text {reg, }}$, see [6] and Flaschka [7]. We also verify that $X$ is the geodesic vector field for a flat Riemannian metric $\Gamma$ on $\mathcal{D}$.

First we rewrite Equation (13) as

$$
T_{(\xi, \eta)} \widehat{\pi}(X(\xi, \eta))=\eta \frac{\partial}{\partial \xi}, \quad \text { for }(\xi, \eta) \in \mathcal{D} .
$$

From the definition of the mapping $F_{Q}(2)$ we get

$$
\mathrm{d} z=\mathrm{d} F_{Q}=\frac{1}{\left(\xi^{n-n_{0}}(1-\xi)^{n-n_{1}}\right)^{1 / n}} \mathrm{~d} \xi=\frac{1}{\eta} \mathrm{d} \xi,
$$

where we use the same complex $n$th root as in the definition of $F_{Q}$. This implies

$$
\frac{\partial}{\partial z}=T_{\xi} F_{Q}\left(\eta \frac{\partial}{\partial \xi}\right), \quad \text { for }(\xi, \eta) \in \mathcal{D}
$$

For each $(\xi, \eta) \in \mathcal{D}$ using (36) and (37) we get

$$
T_{(\xi, \eta)} \delta(X(\xi, \eta))=\left(T_{\xi} F_{Q} \circ T_{(\xi, \eta)} \widehat{\pi}\right)(X(\xi, \eta))=T_{\xi} F_{Q}\left(\eta \frac{\partial}{\partial \xi}\right)=\left.\frac{\partial}{\partial z}\right|_{z=\delta(\xi, \eta)} .
$$

So the holomorphic vector field $X$ (12) on $\mathcal{D}$ and the holomorphic vector field $\frac{\partial}{\partial z}$ on $Q$ are $\delta$-related. Hence $\delta$ sends an integral curve of the vector field $X$ starting at $(\xi, \eta) \in \mathcal{D}$ onto an integral curve of the vector field $\frac{\partial}{\partial z}$ starting at $z=\delta(\xi, \eta) \in Q$. Since an integral curve of $\frac{\partial}{\partial z}$ is a horizontal line segment in $Q$, we have proved

Theorem 5. The holomorphic mapping $\delta$ (35) straightens the holomorphic vector field X (12) on the fundamental domain $\mathcal{D} \subseteq \mathcal{S}_{\text {reg. }}$.

We can say more. Let $u=\operatorname{Re} z$ and $v=\operatorname{Im} z$. Then

$$
\gamma=\mathrm{d} u \odot \mathrm{d} u+\mathrm{d} v \odot \mathrm{d} v=\mathrm{d} z \odot \overline{\mathrm{d} z}
$$

is the flat Euclidean metric on $\mathbb{C}$. Its restriction $\gamma|\mathbb{C}| \mathbb{V}^{+}$to $\mathbb{C} \backslash \mathbb{V}^{+}$is invariant under the group $\mathfrak{G}$, which is a subgroup of the Euclidean group $\mathrm{E}(2)$.

Consider the flat Riemannian metric $\left.\gamma\right|_{Q}$ on $Q$, where $\gamma$ is the metric (38) on $\mathbb{C}$. Pulling back $\left.\gamma\right|_{Q}$ by the mapping $F_{Q}(2)$ gives a metric

$$
\widetilde{\gamma}=F_{Q}^{*}\left(\left.\gamma\right|_{Q}\right)=\left|\xi^{n-n_{0}}(1-\xi)^{n-n_{1}}\right|^{-2 / n} \mathrm{~d} \xi \odot \overline{\mathrm{d}} \xi
$$


on $\mathbb{C} \backslash\{0,1\}$. Pulling the metric $\tilde{\gamma}$ back by the projection mapping $\tilde{\pi}: \mathbb{C}^{2} \rightarrow \mathbb{C}:(\xi, \eta) \mapsto \xi$ gives

$$
\widetilde{\Gamma}=\tilde{\pi}^{*} \widetilde{\gamma}=\left|\xi^{n-n_{0}}(1-\xi)^{n-n_{1}}\right|^{-2 / n} \mathrm{~d} \xi \odot \overline{\mathrm{d} \xi}
$$

on $\mathbb{C}^{2}$. Restricting $\widetilde{\Gamma}$ to the affine Riemann surface $\mathcal{S}_{\text {reg }}$ gives $\Gamma=\frac{1}{\eta} \mathrm{d} \xi \odot \frac{1}{\bar{\eta}} \overline{\mathrm{d} \xi}$.

Lemma 15. $\Gamma$ is a flat Riemannian metric on $\mathcal{S}_{\text {reg. }}$

Proof. We compute. For every $(\xi, \eta) \in \mathcal{S}_{\text {reg }}$ we have

$$
\begin{aligned}
& \Gamma(\xi, \eta)(X(\xi, \eta), X(\xi, \eta))= \\
& \quad=\frac{1}{\eta} \mathrm{d} \xi\left(\eta \frac{\partial}{\partial \xi}+\frac{n-n_{0}}{n} \frac{\xi(1-\xi)\left(1-\frac{2 n-n_{0}-n_{1}}{n} \xi\right)}{\eta^{n-2}} \frac{\partial}{\partial \eta}\right) \cdot \frac{1}{\bar{\eta}} \overline{\mathrm{d}}\left(\bar{\eta} \frac{\partial}{\partial \xi}+\frac{n-n_{0}}{n} \frac{\bar{\xi}(1-\xi)\left(1-\frac{2 n-n_{0}-n_{1}}{n} \tilde{\xi}\right)}{\bar{\eta}^{n-2}} \frac{\partial}{\partial \eta}\right) \\
& \quad=\frac{1}{\eta} \mathrm{d} \xi\left(\eta \frac{\partial}{\partial \xi}\right) \cdot \frac{1}{\bar{\eta}} \overline{\mathrm{d}}\left(\bar{\eta} \overline{\frac{\partial}{\partial \xi}}\right)=1 .
\end{aligned}
$$

Thus, $\Gamma$ is a Riemannian metric on $\mathcal{S}_{\text {reg. }}$ It is flat by construction.

Because $\mathcal{D}$ has nonempty interior and the map $\delta(35)$ is holomorphic, it can be analytically continued to the map

$$
\delta_{Q}: \mathcal{S}_{\text {reg }} \subseteq \mathbb{C}^{2} \rightarrow Q \subseteq \mathbb{C}:(\xi, \eta) \mapsto F_{Q}(\widehat{\pi}(\xi, \eta)),
$$

since $\delta=\left.\delta_{Q}\right|_{\mathcal{D}}$. By construction $\delta_{Q}^{*}\left(\left.\gamma\right|_{Q}\right)=\Gamma$. So the mapping $\delta_{Q}$ is an isometry of $\left(\mathcal{S}_{\mathrm{reg}}, \Gamma\right)$ onto $\left(Q,\left.\gamma\right|_{Q}\right)$. In particular, the map $\delta$ is an isometry of $\left(\mathcal{D},\left.\Gamma\right|_{\mathcal{D}}\right)$ onto $\left(Q,\left.\gamma\right|_{Q}\right)$. Moreover, $\delta$ is a local holomorphic diffeomorphism, because for every $(\xi, \eta) \in \mathcal{D}$, the complex linear mapping $T_{(\xi, \eta)} \delta$ is an isomorphism, since it sends $X(\xi, \eta)$ to $\left.\frac{\partial}{\partial z}\right|_{z=\delta(\xi, \eta)}$ Thus, $\delta$ is a developing map in the sense of differential geometry, see Spivak ([8], p. 97) note on $\S 12$ of Gauss [9]. The map $\delta$ is local because the integral curves of $\frac{\partial}{\partial z}$ on $Q$ are only defined for a finite time, since they are horizontal line segments in $Q$. Thus, the integral curves of $X(12)$ on $\mathcal{D}$ are defined for a finite time. Since the integral curves of $\frac{\partial}{\partial z}$ are geodesics on $\left(Q,\left.\gamma\right|_{Q}\right)$, the image of a local integral curve of $\frac{\partial}{\partial z}$ under the local inverse of the mapping $\delta$ is a local integral curve of $X$. This latter local integral curve is a geodesic on $\left(\mathcal{D},\left.\Gamma\right|_{\mathcal{D}}\right)$, since $\delta$ is an isometry. Thus, we have proved

Theorem 6. The holomorphic vector field X (12) on the fundamental domain $\mathcal{D}$ is the geodesic vector field for the flat Riemannian metric $\left.\Gamma\right|_{\mathcal{D}}$ on $\mathcal{D}$.

Corollary 13. The holomorphic vector field X on the affine Riemann surface $\mathcal{S}_{\text {reg }}$ is the geodesic vector field for the flat Riemannian metric $\Gamma$ on $\mathcal{S}_{\text {reg. }}$.

Proof. The corollary follows by analytic continuation from the conclusion of Theorem 6, since int $\mathcal{D}$ is a nonempty open subset of $\mathcal{S}_{\text {reg }}$ and both the vector field $X$ and the Riemannian metric $\Gamma$ are holomorphic on $\mathcal{S}_{\text {reg. }}$

\section{Discrete Symmetries and Billiard Motions}

Let $\mathcal{G}$ be the group of homeomorphisms of the affine Riemann surface $\mathcal{S}$ (3) generated by the mappings

$$
\mathcal{R}: \mathcal{S} \rightarrow \mathcal{S}:(\xi, \eta) \mapsto\left(\xi, \mathrm{e}^{2 \pi i / n} \eta\right) \text { and } \mathcal{U}: \mathcal{S} \rightarrow \mathcal{S}:(\xi, \eta) \mapsto(\bar{\xi}, \bar{\eta})
$$

Clearly, the relations $\mathcal{R}^{n}=\mathcal{U}^{2}=e$ hold. For every $(\xi, \eta) \in \mathcal{S}$ we have

$$
\mathcal{U} \mathcal{R}^{-1}(\xi, \eta)=\mathcal{U}\left(\xi, \mathrm{e}^{-2 \pi i / n} \eta\right)=\left(\bar{\zeta}, \mathrm{e}^{2 \pi i / n} \bar{\eta}\right)=\mathcal{R}(\bar{\zeta}, \bar{\eta})=\mathcal{R} \mathcal{U}(\xi, \eta)
$$

So the additional relation $\mathcal{U} \mathcal{R}^{-1}=\mathcal{R} \mathcal{U}$ holds. Thus, $\mathcal{G}$ is isomorphic to the dihedral group. 
Lemma 16. $\mathcal{G}$ is a group of isometries of $\left(\mathcal{S}_{\text {reg }}, \Gamma\right)$.

Proof. For every $(\xi, \eta) \in \mathcal{S}_{\text {reg we get }}$

$$
\begin{gathered}
\mathcal{R}^{*} \Gamma(\xi, \eta)(X(\xi, \eta), X(\xi, \eta))=\Gamma(\mathcal{R}(\xi, \eta))\left(T_{(\xi, \eta)} \mathcal{R}(X(\xi, \eta)), T_{(\xi, \eta)} \mathcal{R}(X(\xi, \eta))\right) \\
=\Gamma\left(\xi, \mathrm{e}^{2 \pi i / n} \eta\right)\left(\mathrm{e}^{2 \pi i / n} \eta \frac{\partial}{\partial \xi}+\frac{n-n_{0}}{n} \frac{\xi(1-\xi)\left(1-\frac{2 n-n_{0}-n_{1}}{n-n_{0}} \xi\right)}{\eta^{n-2}} \mathrm{e}^{2 \pi i / n} \frac{\partial}{\partial \eta}\right. \\
\left.\mathrm{e}^{2 \pi i / n} \eta \frac{\partial}{\partial \xi}+\frac{n-n_{0}}{n} \frac{\xi(1-\xi)\left(1-\frac{2 n-n_{0}-n_{1}}{n-n_{0}} \xi\right)}{\eta^{n-2}} \mathrm{e}^{2 \pi i / n} \frac{\partial}{\partial \eta}\right) \\
=\frac{1}{\left|\mathrm{e}^{2 \pi i / n} \eta\right|^{2}} \mathrm{~d} \xi\left(\mathrm{e}^{2 \pi i / n} \eta \frac{\partial}{\partial \xi}\right) \cdot \overline{\mathrm{d} \xi}\left(\mathrm{e}^{2 \pi i / n} \eta \frac{\partial}{\partial \xi}\right)=1 \\
=\frac{1}{|\eta|^{2}} \mathrm{~d} \xi\left(\eta \frac{\partial}{\partial \xi}\right) \cdot \overline{\mathrm{d} \xi}\left(\eta \frac{\partial}{\partial \xi}\right)=\Gamma(\xi, \eta)(X(\xi, \eta), X(\xi, \eta))
\end{gathered}
$$

and

$$
\begin{aligned}
& \mathcal{U}^{*} \Gamma(\xi, \eta)(X(\xi, \eta), X(\xi, \eta))=\Gamma(\mathcal{U}(\xi, \eta))\left(T_{(\xi, \eta)} \mathcal{U}(X(\xi, \eta)), T_{(\xi, \eta)} \mathcal{U}(X(\xi, \eta))\right)
\end{aligned}
$$

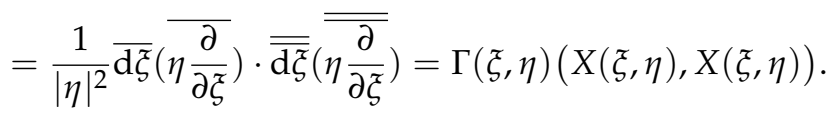

Recall that the group $G$, generated by the linear mappings

$$
R: \mathbb{C} \rightarrow \mathbb{C}: z \mapsto \mathrm{e}^{2 \pi i / n} z \text { and } U: \mathbb{C} \rightarrow \mathbb{C}: z \mapsto \bar{z},
$$

is isomorphic to the dihedral group.

Lemma 17. $G$ is a group of isometries of $(\mathbb{C}, \gamma)$.

Proof. This follows because $R$ and $U$ are Euclidean motions.

We would like the developing map $\delta_{Q}(39)$ to intertwine the actions of $\mathcal{G}$ and $G$ and the geodesic flows on $\left(\mathcal{S}_{\text {reg }}, \Gamma\right)$ and $\left(Q,\left.\gamma\right|_{Q}\right)$. There are several difficulties. The first is: the group $G$ does not preserve the quadrilateral $Q$. To overcome this difficulty we extend the mapping $\delta_{Q}(39)$ to the mapping $\delta_{K^{*}}(17)$ of the affine Riemann surface $\mathcal{S}_{\text {reg }}$ onto the regular stellated $n$-gon $K^{*}$.

Lemma 18. The mapping $\delta_{K^{*}}(17)$ intertwines the action $\Phi(14)$ of $\mathcal{G}$ on $\mathcal{S}_{\text {reg }}$ with the action

$$
\Psi: G \times K^{*} \rightarrow K^{*}:(g, z) \mapsto g(z)
$$

of $G$ on the regular stellated n-gon $K^{*}$.

Proof. From the definition of the mapping $\delta_{K^{*}}$ we see that for each $(\xi, \eta) \in \mathcal{D}$ we have $\delta_{K^{*}}\left(\mathcal{R}^{j}(\xi, \eta)\right)=R^{j} \delta_{K^{*}}(\xi, \eta)$ for every $j \in \mathbb{Z}$. By analytic continuation we see that the preceding equation holds for every $(\xi, \eta) \in \mathcal{S}_{\text {reg. Since }} F_{Q}(\bar{\xi})=\overline{F_{Q}(\xi)}$ by construction and $\widehat{\pi}(\bar{\xi}, \bar{\eta})=\bar{\xi}(11)$, from the definition of the mapping $\delta(35)$ we get $\delta(\bar{\xi}, \bar{\eta})=\overline{\delta(\xi, \eta)}$ for every $(\xi, \eta) \in \mathcal{D}$. In other words, $\delta_{K^{*}}(\mathcal{U}(\xi, \eta))=\mathcal{U} \delta_{K^{*}}(\xi, \eta)$ for every $(\xi, \eta) \in \mathcal{D}$. By analytic continuation we see that the preceding equation holds for all $(\xi, \eta) \in \mathcal{S}_{\text {reg. }}$. Hence on $\mathcal{S}_{\text {reg }}$ we have

$$
\delta_{K^{*} \circ} \Phi_{g}=\Psi_{\varphi(g)} \circ \delta_{K^{*}} \quad \text { for every } g \in \mathcal{G} .
$$

The mapping $\varphi: \mathcal{G} \rightarrow G$ sends the generators $\mathcal{R}$ and $\mathcal{U}$ of the group $\mathcal{G}$ to the generators $R$ and $U$ of the group $G$, respectively. So it is an isomorphism. 
There is a second more serious difficulty: the integral curves of $\frac{\partial}{\partial z}$ run off the quadrilateral $Q$ in finite time. We fix this by requiring that when an integral curve reaches a point $P$ on the boundary $\partial Q$ of $Q$, which is not a vertex, it undergoes a specular reflection at $P$. (If the integral curve reaches a vertex of $Q$ in forward or backward time, then the motion ends). This motion can be continued as a straight line motion, which extends the motion on the original segment in $Q$.

To make this precise, we give $Q$ the orientation induced from $\mathbb{C}$ and suppose that the incoming (and hence outgoing) straight line motion has the same orientation as $\partial Q$. If the incoming motion makes an angle $\alpha$ with respect to the inward pointing normal $N$ to $\partial Q$ at $P$, then the outgoing motion makes an angle $\alpha$ with the normal $N$, see Richens and Berry [2]. Specifically, if the incoming motion to $P$ is an integral curve of $\frac{\partial}{\partial z}$, then the outgoing motion, after reflection at $P$, is an integral curve of $R^{-1} \frac{\partial}{\partial z}=\mathrm{e}^{-2 \pi i / n} \frac{\partial}{\partial z}$. Thus, the outward motion makes a turn of $-2 \pi / n$ at $P$ towards the interior of $Q$, see Figure 10 (left). In Figure 10 (right) the incoming motion has the opposite orientation from $\partial Q$. This extended motion on $Q$ is called a billiard motion. A billiard motion starting in the interior of $\operatorname{cl}(Q) \backslash(\operatorname{cl}(Q) \cap \mathbb{R})$ is defined for all time and remains in $\operatorname{cl}(Q)$ less its vertices, since each of the segments of the billiard motion is a straight line parallel to an edge of $\operatorname{cl}(Q)$ and does not hit a vertex of $\mathrm{cl}(Q)$, see Figure 11 .
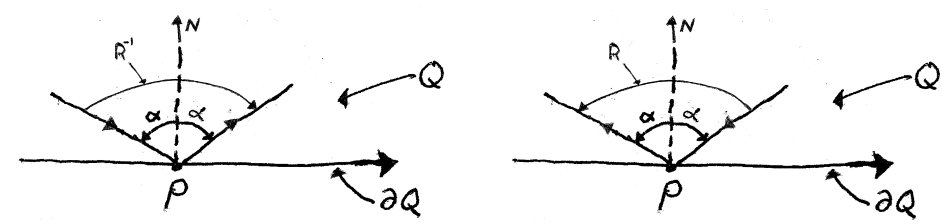

Figure 10. Reflection at a point $P$ on $\partial Q$.
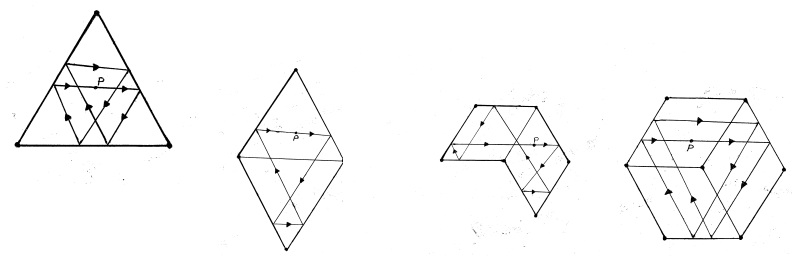

Figure 11. A periodic billiard motion in the equilateral triangle $T=T_{1,1,1}$ starting at $P$. First, extended by the reflection $U$ to a periodic billiard motion in the quadrilateral $Q=T \cup U(T)$. Second, extended by the relection $S$ to a periodic billiard motion in $Q \cup S(Q)$. Third, extended by the reflection $S R$ to a periodic billiard motion in the stellated equilateral triangle $H=K_{1,1,1}^{*}=Q \cup S(Q) S R(S(Q))$.

We can do more. If we apply a reflection $S$ in the edge of $Q$ in its boundary $\partial Q$, which contains the reflection point $P$, to the initial reflected motion at $P$, see Figure 12.

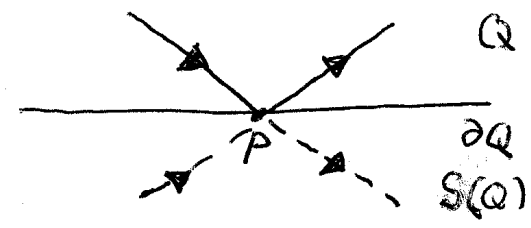

Figure 12. Continuation of a billiard motion in the quadrilateral $Q$ to a billiard motion in the quarilateral $S(Q)$ obtained by the reflection $S$ in an edge of $Q$.

The motion in $S(Q)$ when it reaches $\partial S(Q)$, et cetera, the extended motion becomes a billiard motion in the regular stellated $n$-gon $\left.K^{*}=Q \cup \amalg_{0 \leq k \leq n-1} S R^{k}(Q)\right)$, see Figure 11 . So we have verified 
Theorem 7. A billiard motion in the regular stellated n-gon $K^{*}$, which starts at a point in the interior of $K^{*} \backslash \mathrm{O}$ and does not hit a vertex of $\mathrm{cl}\left(K^{*}\right)$, is invariant under the action of the isometry subgroup $\widehat{G}$ of the isometry group $G$ of $\left(K^{*},\left.\gamma\right|_{K^{*}}\right)$ generated by the rotation $R$.

Let $\widehat{\mathcal{G}}$ be the subgroup of $\mathcal{G}$ generated by the rotation $\mathcal{R}$. We now show

Lemma 19. The holomorphic vector field $X(12)$ on $\mathcal{S}_{\text {reg }}$ is $\widehat{\mathcal{G}}$-invariant.

Proof. We compute. For every $(\xi, \eta) \in \mathcal{S}_{\text {reg }}$ and for $\mathcal{R} \in \widehat{\mathcal{G}}$ we have

$$
\begin{aligned}
T_{(\xi, \eta)} \Phi_{\mathcal{R}}(X(\xi, \eta))=\mathrm{e}^{2 \pi i / n}\left[\eta \frac{\partial}{\partial \xi}+\frac{n-n_{0}}{n} \frac{\xi(1-\xi)\left(1-\frac{2 n-n_{0}-n_{1}}{n} \xi\right)}{\eta^{n-2}} \frac{\partial}{\partial \eta}\right] \\
=\left(\mathrm{e}^{2 \pi i / n} \eta\right) \frac{\partial}{\partial \xi}+\frac{n-n_{0}}{n} \frac{\xi(1-\xi)\left(1-\frac{2 n-n_{0}-n_{1}}{n} \xi\right)}{\left(\mathrm{e}^{2 \pi i / n} \eta\right)^{n-2}} \frac{\partial}{\partial\left(\mathrm{e}^{2 \pi i / n} \eta\right)} \\
=X\left(\xi, \mathrm{e}^{2 \pi i / n} \eta\right)=X_{\circ} \Phi_{\mathcal{R}}(\xi, \eta) .
\end{aligned}
$$

Hence for every $j \in \mathbb{Z}$ we get

$$
T_{(\xi, \eta)} \Phi_{\mathcal{R}^{j}}(X(\xi, \eta))=X \circ \Phi_{\mathcal{R}^{j}}(\xi, \eta)
$$

for every $(\xi, \eta) \in \mathcal{S}_{\text {reg. }}$. In other words, the vector field $X$ is invariant under the action of $\widehat{\mathcal{G}}$ on $\mathcal{S}_{\text {reg. }}$.

Corollary 14. For every $(\xi, \eta) \in \mathcal{D}$ we have

$$
\left.X\right|_{\Phi_{\mathcal{R}^{j}}(\mathcal{D})}=\left.T \Phi_{\mathcal{R}^{j}} X\right|_{\mathcal{D}}
$$

Proof. Equation (43) is a rewrite of Equation (42).

Corollary 15. Every geodesic on $\left(\mathcal{S}_{\text {reg }}, \Gamma\right)$ is $\widehat{\mathcal{G}}$-invariant.

Proof. This follows immediately from the lemma.

Lemma 20. For every $(\xi, \eta) \in \mathcal{S}_{\text {reg }}$ and every $j \in \mathbb{Z}$ we have

$$
T_{\Phi_{\mathcal{R}^{j}}(\xi, \eta)} \delta_{K^{*}}(X(\xi, \eta))=\left.\frac{\partial}{\partial z}\right|_{\delta_{K^{*}}\left(\Phi_{\mathcal{R}^{j}}(\xi, \eta)\right)=R^{j}} j_{z .}
$$

Proof. From Equation (41) we get $\delta_{K^{*} \circ} \Phi_{\mathcal{R}}=\Psi_{R^{\circ}} \delta_{K^{*}}$ on $\mathcal{S}_{\text {reg. }}$. Differentiating the preceding equation and then evaluating the result at $X(\xi, \eta) \in T_{(\xi, \eta)} \mathcal{S}_{\text {reg }}$ gives

$$
\left(T_{\Phi_{\mathcal{R}}(\xi, \eta)} \delta_{K^{*} \circ} T_{(\xi, \eta)} \Phi_{\mathcal{R}}\right) X(\xi, \eta)=\left(T_{\delta_{K^{*}}(\xi, \eta)} \Psi_{R^{\circ}} T_{(\xi, \eta)} \delta_{K^{*}}\right) X(\xi, \eta)
$$

for all $(\xi, \eta) \in \mathcal{S}_{\text {reg. }}$. When $(\xi, \eta) \in \mathcal{D}$, by definition $\delta_{K^{*}}(\xi, \eta)=\delta(\xi, \eta)$. So for every $(\xi, \eta) \in \mathcal{S}_{\text {reg }}$

$$
T_{(\xi, \eta)} \delta_{K^{*}}(X(\xi, \eta))=T_{(\xi, \eta)} \delta(X(\xi, \eta))=\left.\frac{\partial}{\partial z}\right|_{z=\delta(\xi, \eta)}=\left.\frac{\partial}{\partial z}\right|_{z=\delta_{K^{*}}(\xi, \eta)} .
$$

Thus,

$$
T_{\Phi_{\mathcal{R}}(\xi, \eta)} \delta_{K^{*}}\left(T_{(\xi, \eta)} \Phi_{\mathcal{R}} X(\xi, \eta)\right)=T_{\delta_{K^{*}}(\xi, \eta)} \Psi_{R}\left(\left.\frac{\partial}{\partial z}\right|_{z=\delta_{K^{*}}(\xi, \eta)}\right),
$$

for every $(\xi, \eta) \in \mathcal{D}$. By analytic continuation (45) holds for every $(\xi, \eta) \in \mathcal{S}_{\text {reg. Now }}$ $T_{(\xi, \eta)} \Phi_{\mathcal{R}}$ sends $T_{(\xi, \eta)} \mathcal{S}_{\text {reg }}$ to $T_{\Phi_{\mathcal{R}}(\xi, \eta)} \mathcal{S}_{\text {reg. }}$. Since $T_{(\xi, \eta)} \Phi_{\mathcal{R}} X(\xi, \eta)=\mathrm{e}^{2 \pi i / n} X(\xi, \eta)$ for every 
$(\xi, \eta) \in \mathcal{S}_{\text {reg, }}$, it follows that $\mathrm{e}^{2 \pi i / n} X(\xi, \eta)$ is in $T_{\Phi_{\mathcal{R}}(\xi, \eta)} \mathcal{S}_{\text {reg. }}$. Furthermore, since $T_{\delta_{K^{*}}(\xi, \eta)} \Psi_{R}$ sends $T_{\delta_{K^{*}}(\xi, \eta)} K^{*}$ to $T_{\Psi_{R}\left(\delta_{K^{*}}(\xi, \eta)\right.} K^{*}$, we get

$$
T_{\delta_{K^{*}}(\xi, \eta)} \Psi_{R}\left(\left.\frac{\partial}{\partial z}\right|_{z=\delta_{K^{*}(\xi, \eta)}}\right)=\left.R \frac{\partial}{\partial z}\right|_{R z=\Psi_{R}\left(\delta_{K^{*}}(\tilde{\xi}, \eta)\right)} .
$$

For every $(\xi, \eta) \in \mathcal{S}_{\text {reg }}$ we obtain

$$
T_{\Phi_{\mathcal{R}}(\xi, \eta)} \delta_{K^{*}}(X(\xi, \eta))=\left.\frac{\partial}{\partial z}\right|_{R z=\Psi_{\mathcal{R}}\left(\delta_{K^{*}}(\xi, \eta)\right)},
$$

that is, Equation (44) holds with $j=0$. A similar calculation shows that Equation (46) holds with $\mathcal{R}$ replaces by $\mathcal{R}^{j}$. This verifies Equation (44).

We now show

Theorem 8. The image of a $\widehat{\mathcal{G}}$ invariant geodesic on $\left(\mathcal{S}_{\text {reg, }}, \Gamma\right)$ under the developing map $\delta_{K^{*}}(17)$ is a billiard motion in $K^{*}$, see Figure 13.
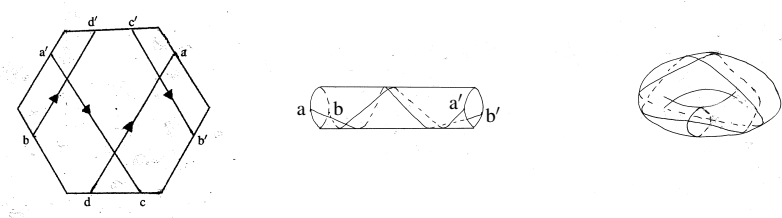

Figure 13. (left) A billiard motion in $K^{*}=K_{1,1,1}^{*}$. (center) The points $c, c^{\prime}$ and $d, d^{\prime}$ in $K^{*}$ are identified, which results in motion on a cylinder. (right) After identifying the points $a, a^{\prime}$ and $b, b^{\prime}$ on the cylinder the motion becomes a periodic geodesic on $\widetilde{S}_{\text {reg }}=\left(K^{*} \backslash\{O\}\right) \sim / G$ on a smooth 2-torus less three points.

Proof. Because $\Phi_{\mathcal{R}^{j}}$ and $\Psi_{R^{j}}$ are isometries of $\left(\mathcal{S}_{\text {reg }}, \Gamma\right)$ and $\left(K^{*},\left.\gamma\right|_{K^{*}}\right)$, respectively, it follows from equation (41) that the surjective map $\delta_{K^{*}}:\left(\mathcal{S}_{\text {reg, }}, \Gamma\right) \rightarrow\left(K^{*},\left.\gamma\right|_{K^{*}}\right)(17)$ is an isometry. Hence $\delta_{K^{*}}$ is a local developing map. Using the local inverse of $\delta_{K^{*}}$ and Equation (44), it follows that a billiard motion in $\operatorname{int}\left(K^{*} \backslash \mathrm{O}\right)$ is mapped onto a geodesic in $\left(\mathcal{S}_{\text {reg }}, \Gamma\right)$, which is possibly broken at the points $\left(\xi_{i}, \eta_{i}\right)=\delta_{K^{*}}^{-1}\left(p_{i}\right)$. Here $p_{i} \in \partial K^{*}$ are the points where the billiard motion undergoes a reflection. However, the geodesic on $\mathcal{S}_{\text {reg }}$ is smooth at $\left(\xi_{i}, \eta_{i}\right)$ since the geodesic vector field $X$ is holomorphic on $\mathcal{S}_{\text {reg }}$. Thus, the image of the geodesic under the developing map $\delta_{K^{*}}$ is a billiard motion.

Theorem 9. Under the restriction of the mapping

$$
v=\sigma_{\circ} \Pi: \mathbb{C} \backslash \mathbb{V}^{+} \rightarrow\left(\mathbb{C} \backslash \mathbb{V}^{+}\right)^{\sim} / \mathfrak{G}=\widetilde{S}_{\text {reg }}
$$

to $K^{*} \backslash \mathrm{O}$ the image of a billiard motion $\lambda_{z}$ is a smooth geodesic $\widehat{\lambda}_{v(z)}$ on $\left(\widetilde{S}_{\mathrm{reg}}, \widehat{\gamma}\right)$, where $v^{*}(\widehat{\gamma})=$ $\gamma|\mathbb{C}| \mathbb{V}^{+}$.

Proof. Since the Riemannian metric $\gamma$ on $\mathbb{C}$ is invariant under the group of Euclidean motions, the Riemannian metric $\left.\gamma\right|_{K^{*} \backslash \mathrm{O}}$ on $K^{*} \backslash \mathrm{O}$ is $\widehat{G}$-invariant. Hence $\gamma_{K^{*} \backslash \mathrm{O}}$ is invariant under the reflection $S_{m}$ for $m \in\{0,1, \ldots, n-1\}$. So $\left.\gamma\right|_{K^{*} \backslash O}$ pieces together to give a Riemannian metric $\gamma^{\sim}$ on the identification space $\left(K^{*} \backslash O\right)^{\sim}$. In other words, the pull back of $\gamma^{\sim}$ under the map $\left.\Pi\right|_{K^{*} \backslash O}: K^{*} \backslash \mathrm{O} \rightarrow\left(K^{*} \backslash \mathrm{O}\right)^{\sim}$, which identifies equivalent edges of $K^{*}$, is the metric $\left.\gamma\right|_{K^{*} \backslash \mathrm{O}}$. Since $\left.\Pi\right|_{K^{*} \backslash \mathrm{O}}$ intertwines the $G$-action on $K^{*} \backslash \mathrm{O}$ with the $G$-action on $\left(K^{*} \backslash O\right) \sim$, the metric $\gamma^{\sim}$ is $\widehat{G}$-invariant. It is flat because the metric $\gamma$ is flat. So $\gamma^{\sim}$ induces a flat Riemannian metric $\widehat{\gamma}$ on the orbit space $\left(K^{*} \backslash O\right) \sim / G=\widetilde{S}_{\text {reg. }}$. Since the billiard motion $\lambda_{z}$ is a $\widehat{G}$-invariant broken geodesic on $\left(K^{*} \backslash \mathrm{O}, \gamma_{K^{*} \backslash \mathrm{O}}\right)$, it gives rise to a continuous broken 
geodesic $\lambda_{\Pi(z)}^{\sim}$ on $\left(\left(K^{*} \backslash \mathrm{O}\right)^{\sim}, \gamma^{\sim}\right)$, which is $\widehat{G}$-invariant. Thus, $\widehat{\lambda}_{v(z)}=v\left(\lambda_{z}\right)$ is a piecewise smooth geodesic on the smooth $G$-orbit space $\left(\left(K^{*} \backslash O\right)^{\sim} / G=\widetilde{S}_{\text {reg }}, \widehat{\gamma}\right)$.

We need only show that $\widehat{\lambda}_{v(z)}$ is smooth. To see this we argue as follows. Let $s \subseteq K^{*}$ be a closed segment of a billiard motion $\gamma_{z}$, that does not meet a vertex of $\mathrm{cl}\left(K^{*}\right)$. Then $s$ is a horizontal straight line motion in $\mathrm{cl}\left(K^{*}\right)$. Suppose that $E_{k_{0}}$ is the edge of $K^{*}$, perpendicular to the direction $u_{k_{0}}$, which is first met by $s$ and let $P_{k_{0}}$ be the meeting point. Let $S_{k_{0}}$ be the reflection in $E_{k_{0}}$. The continuation of the motion $s$ at $P_{k_{0}}$ is the horizontal line $R S_{k_{0}}(s)$ in $K_{k_{0}}^{*}$. Recall that $K_{k_{0}}^{*}$ is the translation of $K^{*}$ by $\tau_{k_{0}}$. Using a suitable sequence of reflections in the edges of a suitable $K_{k_{0} \cdots k_{\ell}}^{*}$ each followed by a rotation $R$ and then a translation in $\mathcal{T}$ corresponding to their origins, we extend $s$ to a smooth straight line $\lambda$ in $\mathbb{C} \backslash \mathbb{V}^{+}$, see Figure 14. The line $\lambda$ is a geodesic in $\left(\mathbb{C} \backslash \mathbb{V}^{+},\left.\gamma\right|_{\mathbb{C} \backslash \mathbb{V}^{+}}\right)$, which in $K^{*}$ has image $\widehat{\lambda}_{v(z)}$ under the $\mathfrak{G}$-orbit map $v(47)$ that is a smooth geodesic on $\left(\widetilde{S}_{\text {reg, }}, \widehat{\gamma}\right)$. The geodesic $v(\lambda)$ starts at $v(z)$. Thus, the smooth geodesic $v(\lambda)$ and the geodesic $\widehat{\lambda}_{v(z)}$ are equal. In other words, $\widehat{\lambda}_{v(z)}$ is a smooth geodesic.

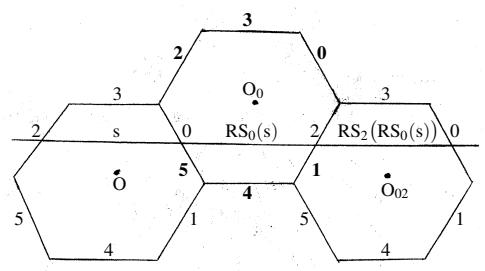

Figure 14. The billiard motion $\gamma_{z}$ in the stellated regular 3-gon $K_{1,1,1}^{*}$ meets the edge 0 , isreflected in this edge by $S_{0}$, and then is rotated by $R$. This gives an extended motion $R S_{0} \gamma_{z}$, which is a straight line that is the same as reflecting $\gamma_{z}$ by $U$ and then translating by $\tau_{0}$.

Thus, the affine orbit space $\widetilde{S}_{\text {reg }}=\left(\mathbb{C} \backslash \mathbb{V}^{+}\right) \sim / \mathfrak{G}$ with flat Riemannian metric $\widehat{\gamma}$ is the affine analogue of the Poincaré model of the affine Riemann surface $\mathcal{S}_{\text {reg }}$ as an orbit space of a discrete subgroup of $\operatorname{PGl}(2, \mathbb{C})$ acting on the unit disk in $\mathbb{C}$ with the Poincare metric.

Funding: This research received no external funding.

Institutional Review Board Statement: Not applicable.

Informed Consent Statement: Not applicable.

Data Availability Statement: No data was used in this research.

Conflicts of Interest: The author declares no conflict of interest.

\section{Appendix A. Group Theoretic Properties}

In this appendix we discuss some group theoretic properties of the set of equivalent edges of $\mathrm{cl}\left(K^{*}\right)$, which we use to determine the topology of $\widetilde{\mathcal{S}}_{\text {reg. }}$.

Let $\mathcal{E}$ be the set of unordered pairs $\left[E, E^{\prime}\right]$ of nonadjacent edges of $\mathrm{cl}\left(K^{*}\right)$. Define an action $\cdot$ of $G$ on $\mathcal{E}$ by

$$
g \cdot\left[E, E^{\prime}\right]=\left[g(E), g\left(E^{\prime}\right)\right]
$$

for every unordered pair $\left[E, E^{\prime}\right]$ of nonadjacent edges of $\mathrm{cl}\left(K^{*}\right)$. For every $g \in G$ the edges $g(E)$ and $g\left(E^{\prime}\right)$ are nonadjacent. This follows because the edges $E$ and $E^{\prime}$ are nonadjacent and the elements of $G$ are invertible mappings of $\mathbb{C}$ into itself. So $\varnothing=g\left(E \cap E^{\prime}\right)=$ $g(E) \cap g\left(E^{\prime}\right)$. Thus, the mapping is well defined. It is an action because for every $g$ and $h \in G$ we have

$$
\begin{aligned}
g \cdot\left(h \cdot\left[E, E^{\prime}\right]\right) & =g \cdot\left[h(E), h\left(E^{\prime}\right)\right]=\left[g \left(h(E), g\left(h\left(E^{\prime}\right)\right]\right.\right. \\
& =\left[(g h)(E),(g h)\left(E^{\prime}\right)\right]=(g h) \cdot\left[E, E^{\prime}\right] .
\end{aligned}
$$


Since $\mathcal{E}=\bigcup_{j=0,1, \infty} \mathcal{E}^{j}$, the action $\cdot$ of $G$ on $\mathcal{E}$ induces an action · of the group $G^{j}$ of reflections on the set $\mathcal{E}^{j}$ of equivalent edges of $\operatorname{cl}\left(K^{*}\right)$, which is defined by

$$
g_{j} \cdot\left[E, S_{k}^{(j)}(E)\right]=\left[g_{j}(E), g_{j}\left(S_{k}^{(j)}(E)\right)\right]=\left[g_{j}(E),\left(g_{j} S_{k}^{(j)} g_{j}^{-1}\right)\left(g_{j}(E)\right)\right],
$$

for every $g_{j} \in G^{j}$, every edge $E$ of $\mathrm{cl}\left(K^{*}\right)$, and every generator $S_{k}^{(j)}$ of $G^{j}$, where $k=0,1, \ldots$, $n-1$. Since $g_{j} S_{k}^{(j)} g_{j}^{-1}=S_{r}^{(j)}$ by Corollary 6 , the mapping $\cdot$ is well defined.

Lemma A1. The group $G$ action $\bullet$ sends a $G^{j}$-orbit on $\mathcal{E}^{j}$ to another $G^{j}$-orbit on $\mathcal{E}^{j}$.

Proof. Consider the $G^{j}$-orbit of $\left[E, S_{m}^{(j)}(E)\right] \in \mathcal{E}^{j}$. For every $g \in G$ we have

$$
g \cdot\left(G^{j} \cdot\left[E, S_{m}^{(j)}(E)\right]\right)=\left(g G^{j} g^{-1}\right) \cdot\left(g \cdot\left[E, S_{m}^{(j)}(E)\right]\right)=G^{j} \cdot\left(g \cdot\left[E, S_{m}^{(j)}(E)\right]\right),
$$

because $G^{j}$ is a normal subgroup of $G$ by Corollary 7. Since

$$
g \cdot\left[E, S_{m}^{(j)}(E)\right]=\left[g(E), g\left(S_{m}^{(j)}(E)\right)\right]=\left[g(E), g S_{m}^{(j)} g^{-1}(g(E))\right]
$$

and $g S_{m}^{(j)} g^{-1}=S_{r}^{(j)}$ by Corollary 6, it follows that $g \bullet\left[E, S_{m}^{(j)}(E)\right] \in \mathcal{E}^{j}$.

Lemma A2. For every $j=0,1, \infty$ and every $k=0,1, \ldots, n-1$ the isotropy group $G_{e_{k}^{j}}^{j}$ of the $G^{j}$ action on $\mathcal{E}^{j}$ at $e_{k}^{j}=\left[E, S_{k}^{(j)}(E)\right]$ is $\left\langle S_{k}^{(j)} \mid\left(S_{k}^{(j)}\right)^{2}=e\right\rangle$.

Proof. Every $g \in G_{e_{k}^{j}}^{j}$ satisfies

$$
e_{k}^{j}=\left[E, S_{k}^{(j)}(E)\right]=g \cdot e_{k}^{j}=g \cdot\left[E, S_{k}^{(j)}(E)\right]
$$

if and only if

$$
\left[E, S_{k}^{(j)}(E)\right]=\left[g(E), g S_{k}^{(j)} g^{-1}(g(E))\right]=\left[g(E), S_{r}^{(j)}(g(E))\right]
$$

if and only if one of the statements 1) $g(E)=E \& S_{k}^{(j)}(E)=S_{r}^{(j)}(g(E))$ or 2) $E=g\left(S_{r}^{(j)}(E)\right)$ $\& g(E)=S_{k}^{(j)}(E)$ holds. From $g(E)=E$ in 1 ) we get $g=e$ using Lemma 3. To see this we argue as follows. If $g \neq e$, then $g=R^{p}\left(S^{(j)}\right)^{\ell}$ for some $\ell=0,1$ and some $p \in\{0,1, \ldots, n-1\}$, see Equation (A1). Suppose that $g=R^{p}$ with $p \neq 0$. Then $g(E) \neq E$, which contradicts our hypothesis. Now suppose that $g=R^{p} S^{(j)}$. Then $E=g(E)=$ $R^{p} S^{(j)}(E)$, which gives $R^{-p}(E)=S^{(j)}(E)$. Let $A$ and $B$ be end points of the edge $E$. Then the reflection $S^{(j)}$ sends $A$ to $B$ and $B$ to $A$, while the rotation $R^{-p}$ sends $A$ to $A$ and $B$ to $B$. Thus, $R^{-p}(E) \neq S^{(j)}(E)$, which is a contradiction. Hence $g=e$. If $g(E)=S_{k}^{(j)}(E)$ in 2), then $\left(S_{k}^{(j)} g\right)(E)=E$. So $S_{k}^{(j)} g=e$ by Lemma 3, that is, $g=S_{k}^{(j)}$.

For every $j=0,1, \infty$ and every $m_{j}=0,1, \ldots, \frac{n}{d_{j}}-1$ let $G_{e_{m_{j} d_{j}}^{j}}=\left\{g_{j} \in G^{j} \mid g_{j}\right.$. $\left.e_{m_{j} d_{j}}^{j}=e_{m_{j} d_{j}}^{j}\right\}$ be the isotropy group of the $G^{j}$ action on $\mathcal{E}^{j}$ at $e_{m_{j} d_{j}}^{j}=\left[E, S_{m_{j} d_{j}}^{(j)}(E)\right]$. Since $G_{e_{m_{j} d_{j}}^{j}}^{j}=\left\langle S_{m_{j} d_{j}}^{(j)} \mid\left(S_{m_{j} d_{j}}^{(j)}\right)^{2}=e\right\rangle$ is an abelian subgroup of $G^{j}$, it is a normal subgroup. Thus, $H^{j}=G^{j} / G_{e_{m_{j} d_{j}}^{j}}^{j}$ is a subgroup of $G^{j}$ of order $\left(2 n / d_{j}\right) / 2=n / d_{j}$. This proves 
Lemma A3. For every $j=0,1, \infty$ and each $m_{j}=0,1, \ldots, \frac{n}{d_{j}}-1$ the $G^{j}$-orbit of $e_{m_{j} d_{j}}$ in $\mathcal{E}^{j}$ is equal to the $H^{j}$-orbit of $e_{m_{j} d_{j}}$ in $\mathcal{E}^{j}$.

Lemma A4. For $j=0,1, \infty$ we have $H^{j}=\left\langle V=R^{d_{j}} \mid V^{n / d_{j}}=e\right\rangle$.

Proof. Since

$$
S_{k}^{(j)}=R^{k} S^{(j)} R^{-k}=R^{k}\left(R^{n_{j}} U\right) R^{-k}=R^{2 k+n_{j}} U=R^{2 k} S^{(j)},
$$

we get $S_{m_{j} d_{j}}^{(j)}=R^{\left(2 m_{j}+\frac{n_{j}}{d_{j}}\right) d_{j}} U=\left(R^{d_{j}}\right)^{m_{j}} S^{(j)}$. Because the group $G^{j}$ is generated by the reflections $S_{k}^{(j)}$ for $k=0,1, \ldots, n-1$, it follows that

$$
G^{j} \subseteq\left\langle V=R^{d_{j}}, S_{m_{j} d_{j}}^{(j)} \mid V^{n / d_{j}}=e=\left(S_{m_{j} d_{j}}^{(j)}\right)^{2} \& V S_{m_{j} d_{j}}^{(j)}=S_{m_{j} d_{j}}^{(j)} V^{-1}\right\rangle=K_{j}
$$

$K_{j}$ is a subgroup of $G$ of order $2 n / d_{j}$. Clearly the isotropy group $G_{e_{m_{j} d_{j}}^{j}}^{j}=\left\langle S_{m_{j} d_{j}}^{(j)}\right|\left(S_{m_{j} d_{j}}^{(j)}\right)^{2}=$ $e\rangle$ is an abelian subgroup of $K^{j}$. Hence $H^{j}=G^{j} / G_{e_{m_{j} d_{j}}^{j}}^{j} \subseteq K^{j} / G_{e_{m_{j} d_{j}}^{j}}=L^{j}$, where $L^{j}$ is a subgroup of $K^{j}$ of order $\left(2 n / d_{j}\right) / 2=n / d_{j}$. Thus, the group $L^{j}$ has the same order as its subgroup $H^{j}$. So $H^{j}=L^{j}$. However, $L^{j}=\left\langle V=R^{d_{j}} \mid V^{n / d_{j}}=e\right\rangle$.

Let $f_{\ell}^{j}=R^{\ell} \cdot e_{0}^{j}$. Then

$$
\begin{aligned}
f_{\ell}^{j} & =R^{\ell} \cdot e_{0}^{j}=R^{\ell} \cdot\left[E, S^{(j)}(E)\right] \\
& =\left[R^{\ell}(E), R^{\ell} S^{(j)} R^{-\ell}\left(R^{\ell}(E)\right)\right]=\left[R^{\ell}(E), S_{\ell}^{(j)}\left(R^{\ell}(E)\right)\right] .
\end{aligned}
$$

So

$$
\begin{aligned}
V^{m} \cdot f_{\ell}^{j} & =V^{m} \cdot\left[R^{\ell}(E), R^{\ell} S^{(j)} R^{-\ell}\left(R^{\ell}(E)\right)\right] \\
& =\left[V^{m}\left(R^{\ell}(E)\right), V^{m} S_{\ell}^{(j)} V^{-m}\left(V^{m}\left(R^{\ell}(E)\right)\right]\right. \\
& =\left[R^{m d_{j}+\ell}(E), S_{m d_{j}+\ell}^{(j)}(E)\right]=e_{m d_{j}+\ell}^{j}
\end{aligned}
$$

This proves

$$
\bigcup_{\ell_{j}=0}^{d_{j}-1} H^{j} \cdot f_{\ell_{j}}^{j}=\bigcup_{\ell_{j}=0}^{d_{j}-1} \bigcup_{m_{j}=0}^{\frac{n}{d_{j}}-1} V^{m_{j}} \cdot f_{\ell_{j}}^{j}=\bigcup_{k=0}^{n-1} e_{k^{\prime}}^{j}
$$

since every $k \in\{0,1, \ldots, n-1\}$ may be written uniquely as $m_{j} d_{j}+\ell_{j}$ for some $m_{j} \in$ $\left\{0,1, \ldots, \frac{n}{d_{j}}-1\right\}$ and some $\ell_{j} \in\left\{0,1, \ldots, d_{j}-1\right\}$.

\section{References}

1. Aurell, E.; Itzykson, C. Rational billiards and algebraic curves. J. Geom. Phys. 1988, 5, 191-208. [CrossRef]

2. Richens, P.J.; Berry, M.V. Pseudointegrable systems in classical and quantum mechanics. Phys. D Nonlinear Phenom. 1981, 2, 495-512. [CrossRef]

3. Berger, M. Geometry Revealed. A Jacob's Ladder to Modern Higher Geometry; Springer: New York, NY, USA, 2010.

4. Weyl, H. The Concept of a Riemann Surface; Dover: Mineola, NY, USA, 2001.

5. $\quad$ McKean, H.; Moll, V. Elliptic Curves; Cambridge University Press: Cambridge, UK, 1999.

6. Bates, L.; Cushman, R. Complete integrability beyond Liouville-Arnol'd. Rep. Math. Phys. 2005, 56, 77-91. [CrossRef]

7. Flaschka, H. A remark on integrable Hamiltonian systems. Phys. Lett. A 1988, 131, 505-508. [CrossRef] 
8. Spivak, M. A Comprehensive Introduction to Differential Geometry; Publish or Perish: Berkeley, CA, USA, 1979 ; Volume 2.

9. Gauss, C.F. Disquisitiones generales circa superficies curvas. In English Translation: General Investigations of Curved Surfaces of 1825 and 1827; Hiltebeitel, A., Morehead, J., Eds.; Princeton University Library: Princeton, NJ, USA, 1902. Available online: www.gutenberg.org/files/36856/36856-pdf.pdf (accessed on 1 February 2020). 